\title{
Synthesis of $\boldsymbol{P}$-Chiral Dihydrobenzooxaphospholes Through Negishi Cross-Coupling
}

Joshua D. Sieber,* Bo Qu, Sonia Rodriguez, Nizar Haddad, Nelu Grinberg, Heewon Lee, Jinhua J. Song, Nathan K. Yee, Chris H. Senanayake

Chemical Development, Boehringer Ingelheim Pharmaceuticals, Inc., 900 Ridgebury Rd., Ridgefield, CT 06877, USA

joshua.sieber@boehringer-ingelheim.com

Supporting Information

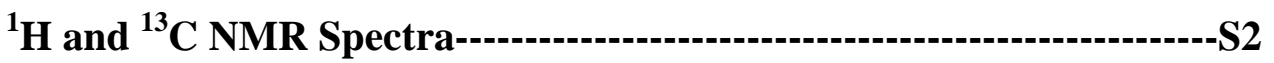




\section{9-003 final}

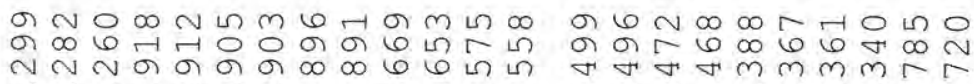

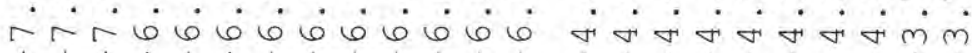

$\$ 11$

$\longrightarrow$

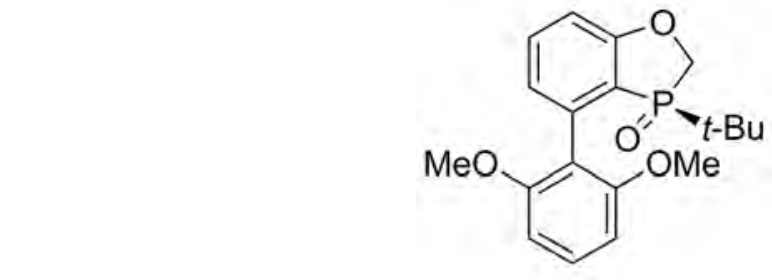

$3 a$
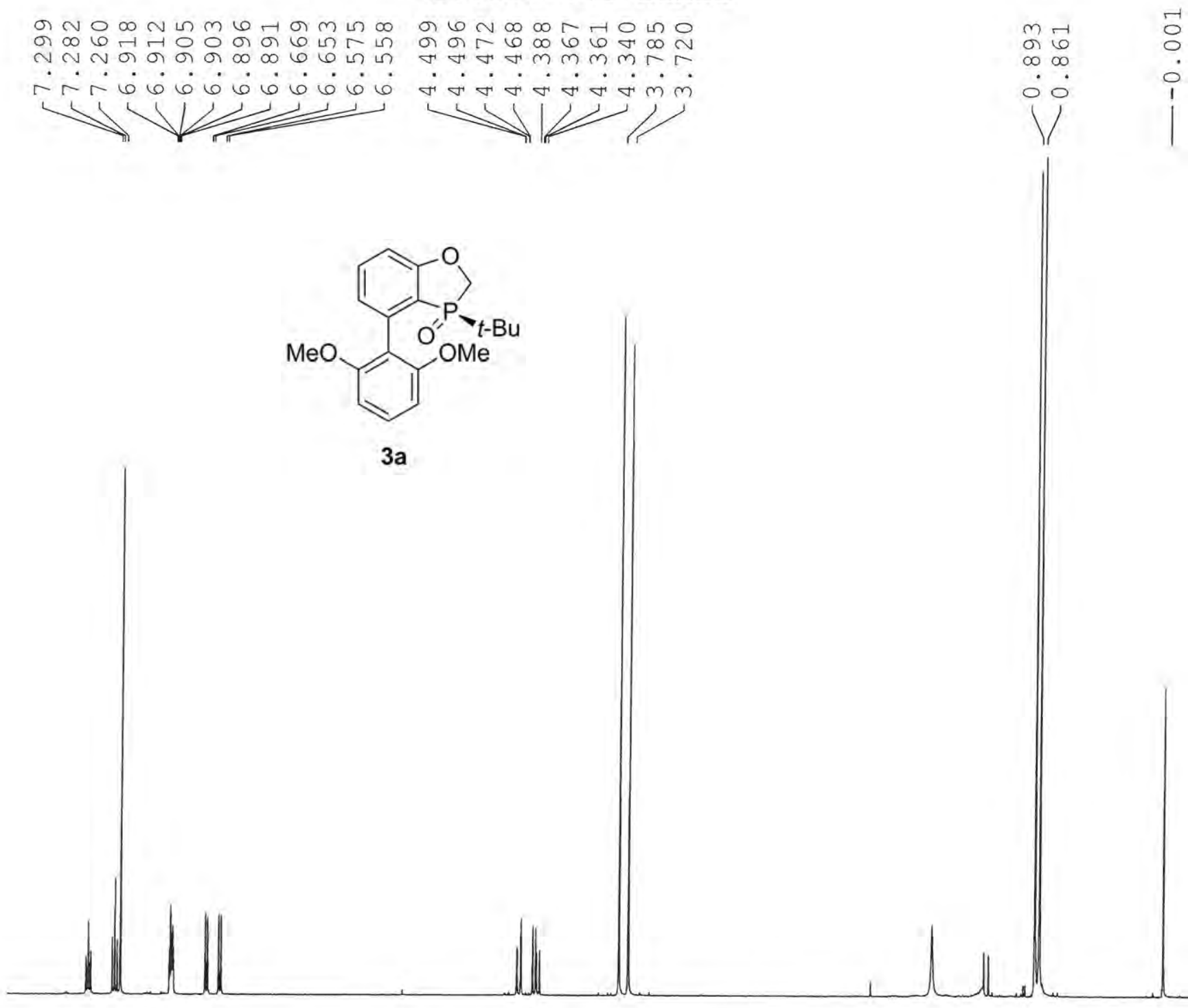

Current Data Parameters

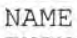

Parameters

EXPNO

5
1

F2 - Acquisition Parameter

Date_ 20150331

Time $\quad 17.50$

INSTRUM

PROBHD

PULPROG

SD

NS

DS

$5 \mathrm{~mm}$ РАВBO BB-

FIDRES $\quad 0.275098 \mathrm{~Hz}$

$\begin{array}{lr}\text { AQ } & 1.8175317 \mathrm{sec} \\ \text { RG } & 512\end{array}$

DW

$D E$

TE

512
55.467 usec

6.50 usec $299.0 \mathrm{~K}$

$2.00000000 \mathrm{sec}$

$=======e$ CHANNEL $\mathrm{f}$

NUC1

P1

PLI $\quad 2.95$ use

PLIW $18.43091774 \mathrm{~W}$

F2 - Processing parameters

$\begin{array}{lr}\text { SI } & 16384 \\ \text { SF } & 500.1300132 \mathrm{MHz}\end{array}$

WDW $\quad 500.1300132$

SSB $\quad 0$

GB

$0.30 \mathrm{~Hz}$

PC

1.00

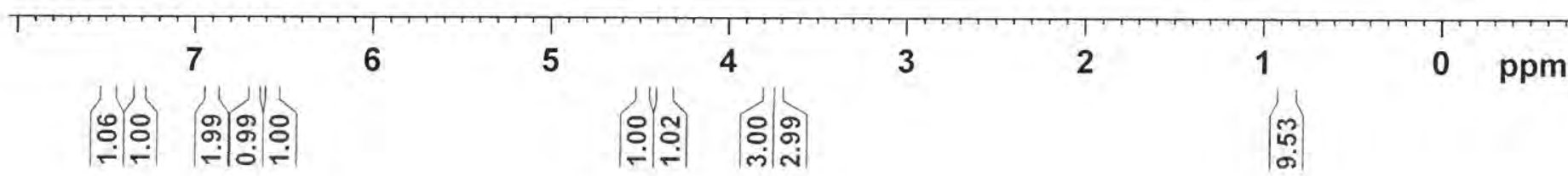




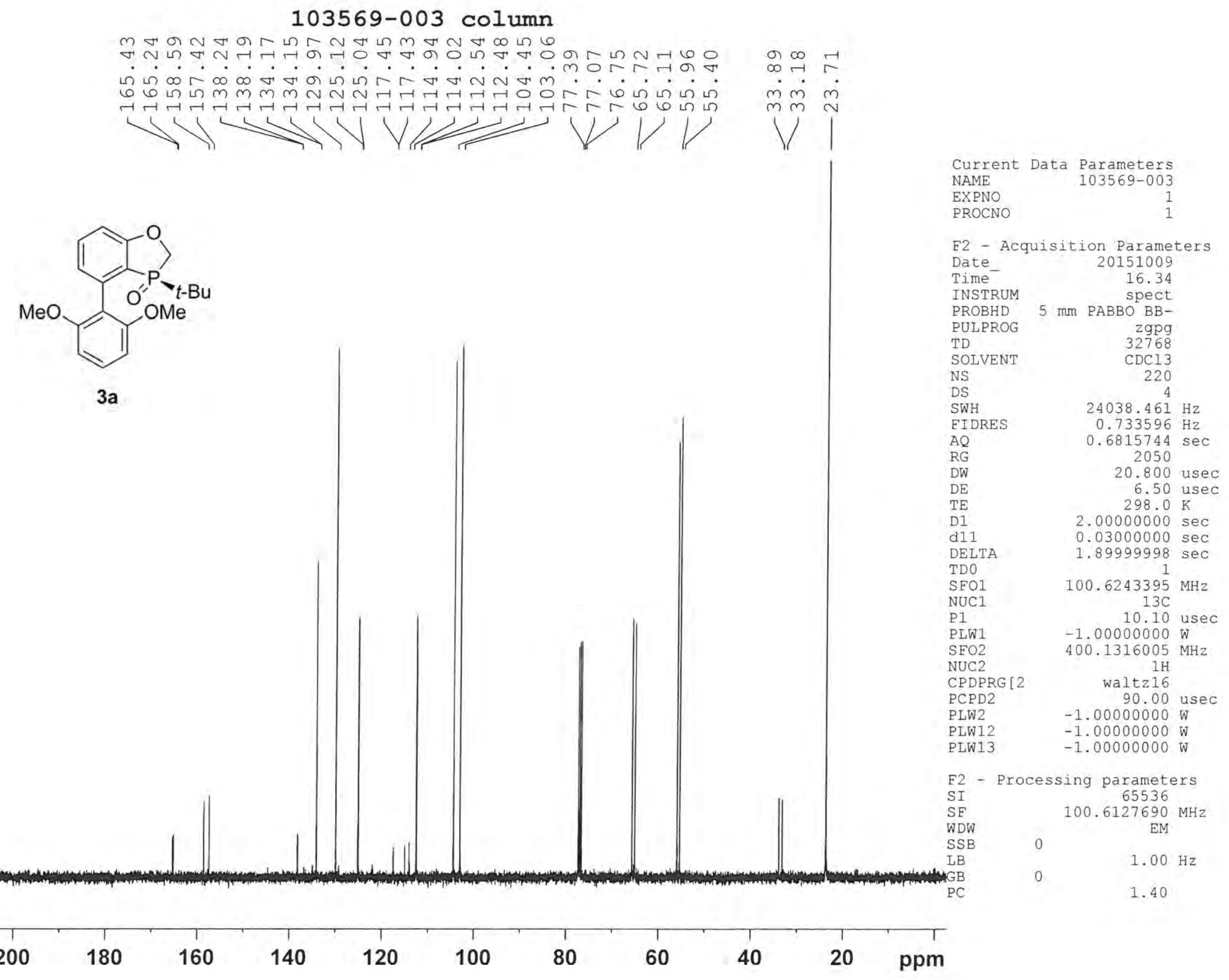




\section{9-057 column}

패 $\infty$ 에 ฟ v

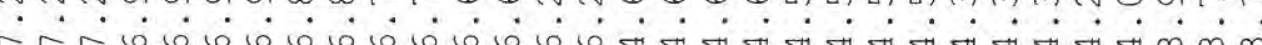

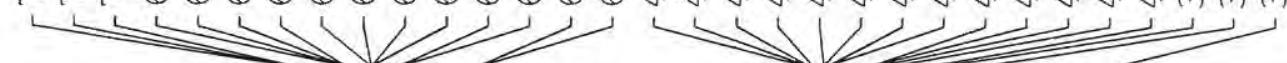

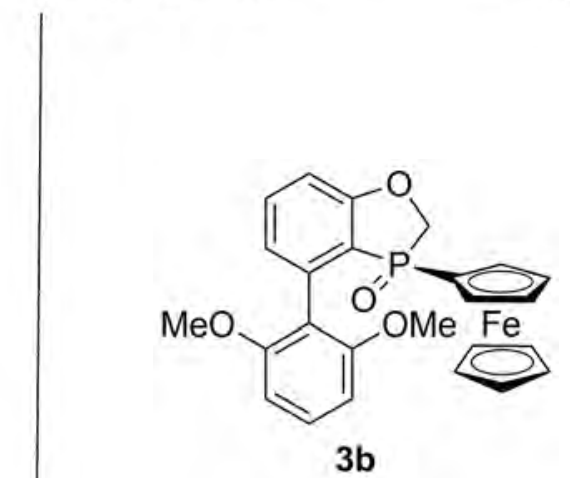

$3 b$

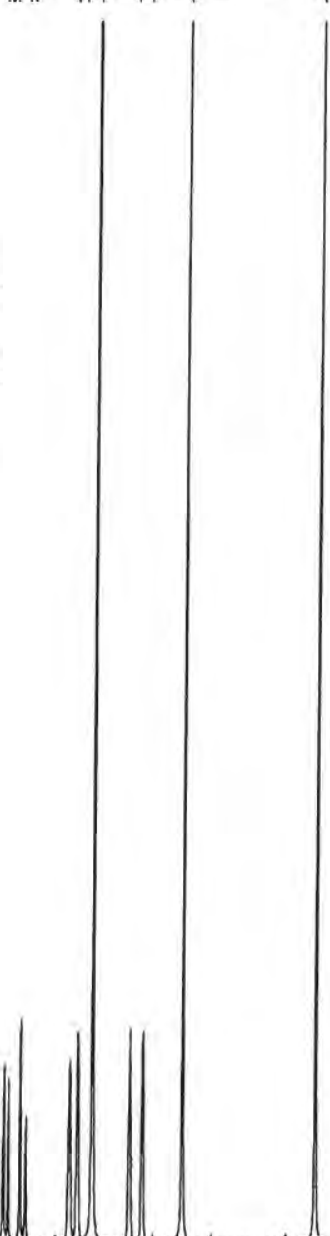

7
0
0
0
1
1

Current Data Parameter NAME

EXPNO

PROCNO

F2 - Acquisition Parameters

Date_ 20151015

Time 11.50

PROBHD $5 \mathrm{~mm}$ PABBO BB-

PULPROG $\quad$ q $\overline{3} 2768$

SOLVENT

DS

SWH

FIDRES

FIDR
AQ
RG

RW

$D E$

TDO

16

$9014.423 \mathrm{~Hz}$

$0.275098 \mathrm{~Hz}$

$1.8175317 \mathrm{sec}$

512

55.467 usec

6.50 usec

$2.00000000 \mathrm{sec}$

$==x==x=$ CHANNEL f $1===x===$ NUC

P1

PLIW $2.10 \mathrm{~dB}$

SEO1 $500.1334009 \mathrm{MHz}$

F2 - Processing parameters

SI 16384

SF $\quad 500.1300134 \mathrm{MHz}$

WDW

SSB

0 EM

$\mathrm{LB}$
$\mathrm{GB}$

PC

$0.30 \mathrm{~Hz}$

1.00

\section{$\begin{array}{llllllllllllllllll}8.5 & 8.0 & 7.5 & 7.0 & 6.5 & 6.0 & 5.5 & 5.0 & 4.5 & 4.0 & 3.5 & 3.0 & 2.5 & 2.0 & 1.5 & 1.0 & 0.5 & \mathrm{ppm}\end{array}$

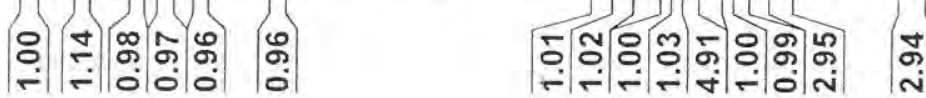




\section{9-057 column}

๒

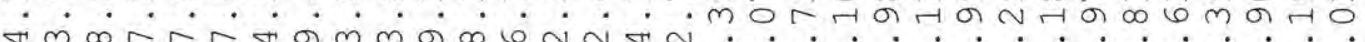

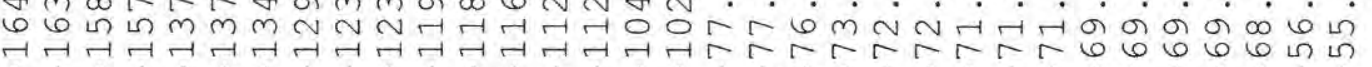
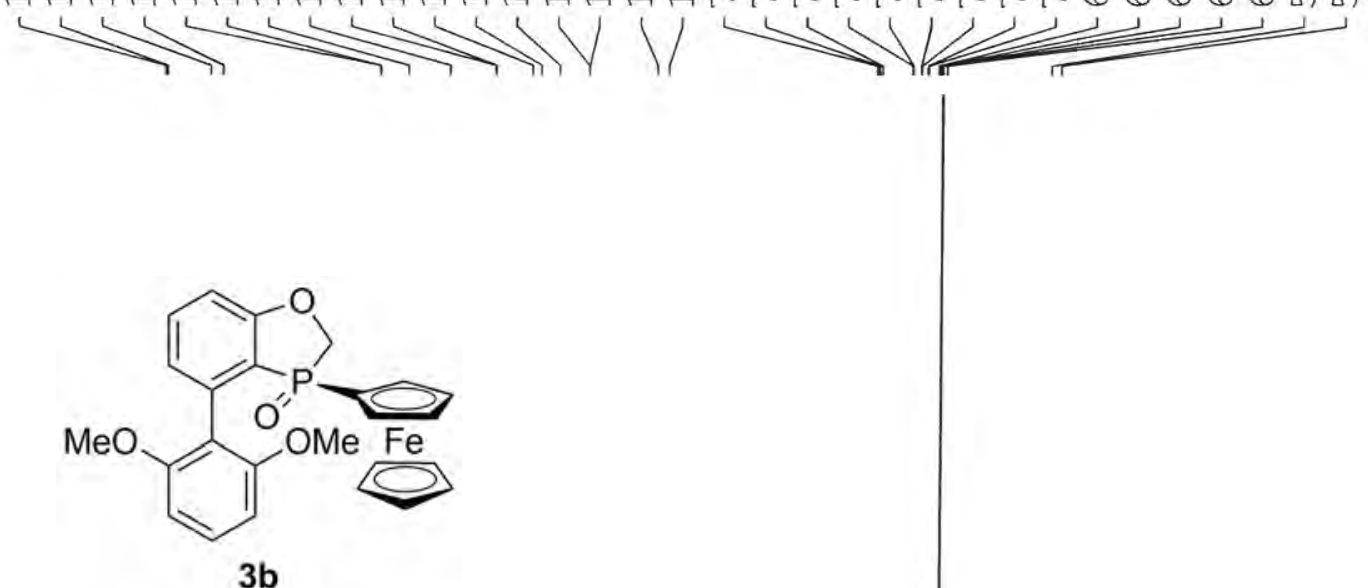

3b

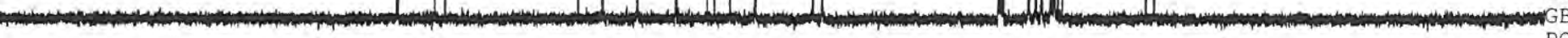

Current Data Parameters NAME 103669-057 EXPNO

F2 - Acquisition Parameters

Date_ 20151014

$\begin{array}{lr}\text { Time } & \\ & \end{array}$

INSTRUM

PROBHD

pect

PULPROG

TD

$5 \mathrm{~mm}$ PABBO BB-

$\begin{array}{lr}\text { SWH } & 24038.461 \mathrm{~Hz} \\ \text { EIDRES } & 0.733596 \mathrm{~Hz}\end{array}$

$0.6815744 \mathrm{sec}$

RG

DW

DE

d11

DELTA

TDO

SFO1

N1

PLW1

$\mathrm{SEO} 2$

CPDPRG [2

PCPD2

PLW2

PLW12

zgpg
32768

$\mathrm{CDCl}$

256
4 2050

20.800 usec 6.50 usec

$2.00000000 \mathrm{sec}$

$0.03000000 \mathrm{sec}$

$1.89999998 \mathrm{sec}$

$100.6243395 \mathrm{MHz}$

$$
13 \mathrm{C}
$$

10.10 usec

$-1.00000000 \mathrm{~W}$

$400.1316005 \mathrm{MHz}$

$$
\text { waltz } 16
$$

90.00 usec

$-1.00000000 \mathrm{~W}$

$-1.00000000 \mathrm{~W}$

F2 - Processing parameters

SI 65536

SF $\quad 100.6127690 \mathrm{MHz}$

SSB $\quad 0$

$100.6127690 \mathrm{MHz}$

$1.00 \mathrm{~Hz}$

1. 40 


\section{9-074 column}

가

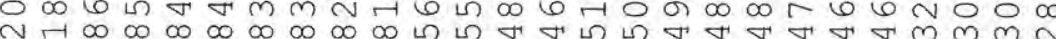

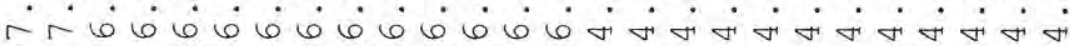
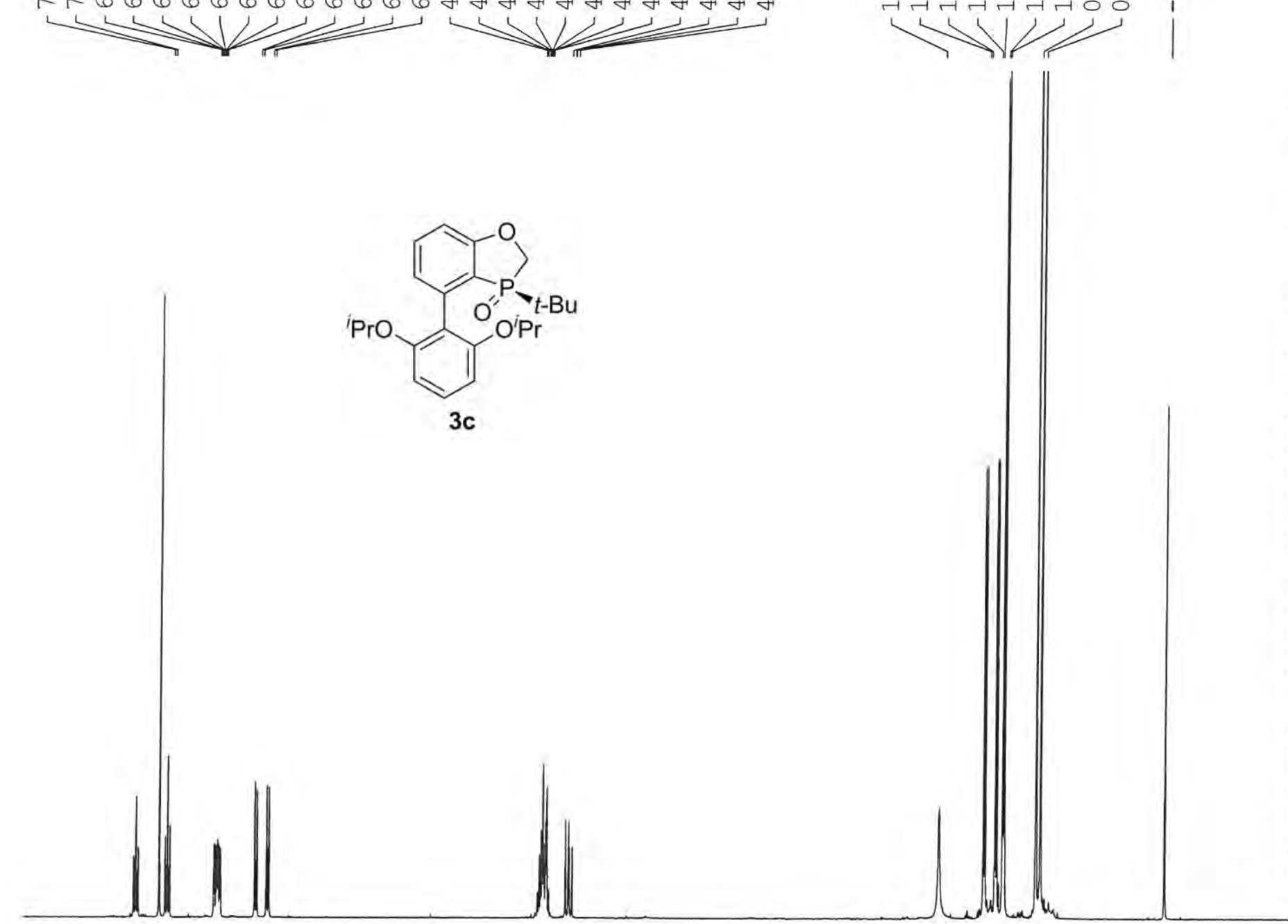

3c

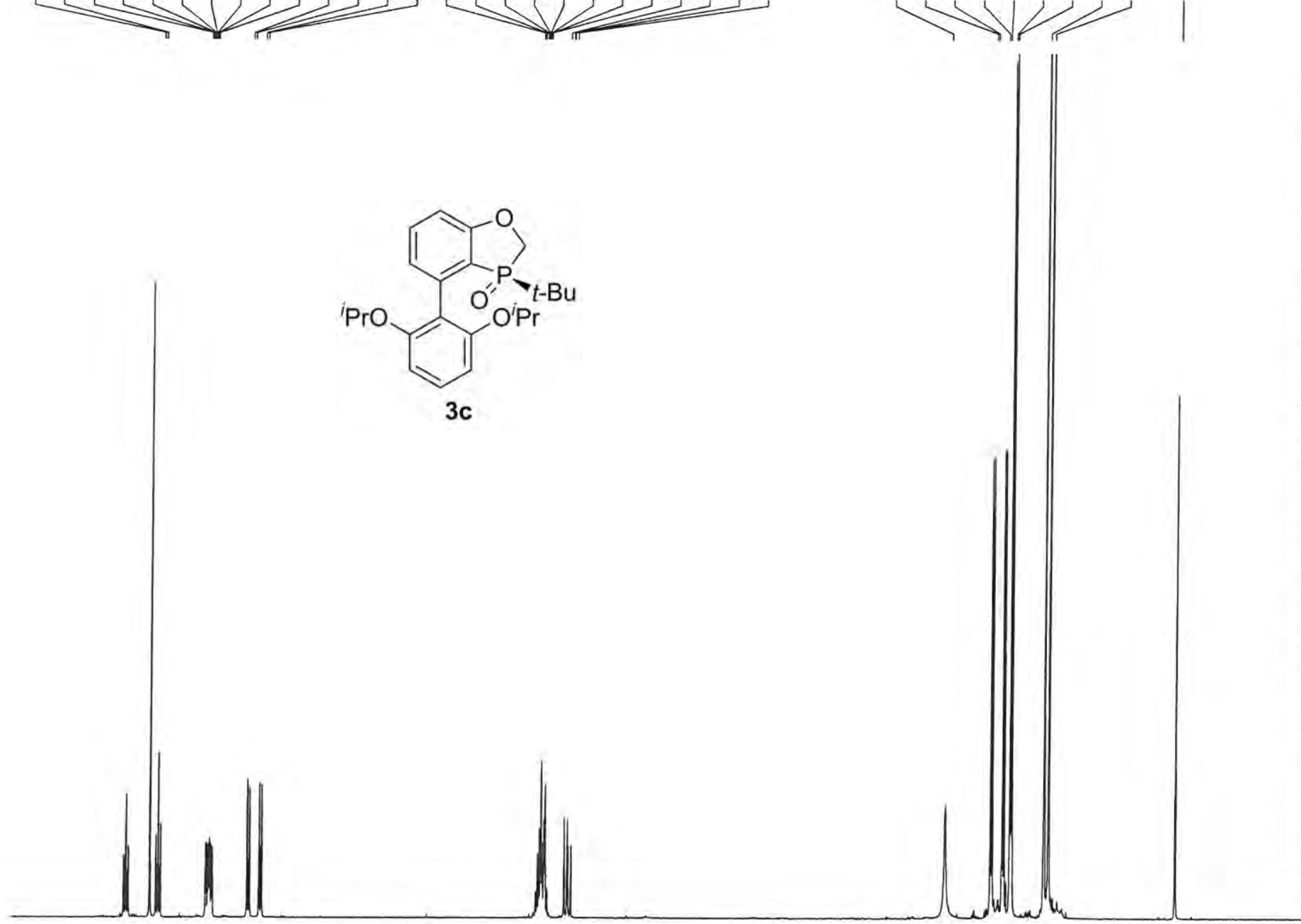

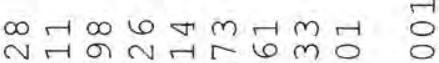

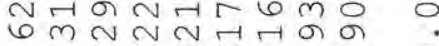

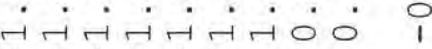

Current Data Parameters $103569-074$ $\operatorname{PROCNO}$

F2 - Acquisition Parameters

Date_rats 2015002

ime $\quad 17.45$

spect

PROBHD $5 \mathrm{~mm}$ PABBI $1 \mathrm{H}-$

PUPROG

SOLVENT

NS

DS

WH

FIDRES

AQ

RG

DW

TE

TDO

$$
\begin{array}{r}
q \quad z g 10 \\
32768 \\
\text { CDC13 } \\
16 \\
0 \\
9014.423 \mathrm{~Hz} \\
0.275098 \mathrm{~Hz} \\
.8175317 \mathrm{sec} \\
1150 \\
55.467 \text { usec } \\
6.50 \text { usec } \\
299.0 \mathrm{~K}
\end{array}
$$

$===-$

NUC1

P1

PL1 1 W

$\mathrm{SFO} 1$

F2 - Processing parameters

SI 16384

SF $\quad 500.1300135 \mathrm{MHz}$

WDW

SSB

GB $\quad 0$

PC . usec

14.981212

$0.30 \mathrm{~Hz}$

1.00
$2.00000000 \mathrm{sec}$

CHANNEL $\mathrm{f}]$

$=======+$

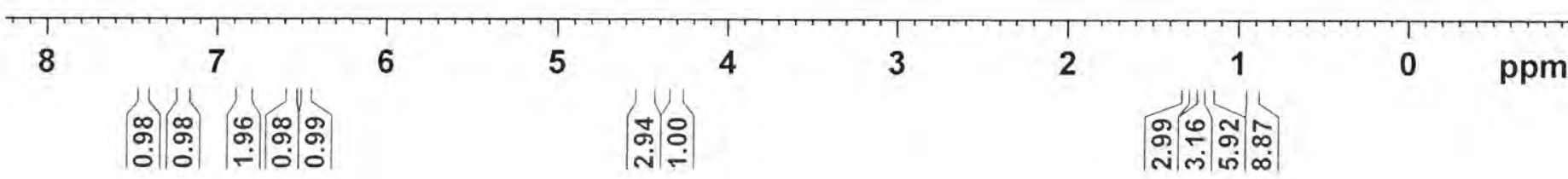




\section{9-038 column}

m $6 m m \infty \pi-6 \pi+N G \pi+\infty \pi \omega$

HLO

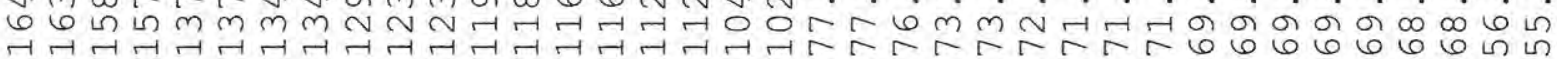
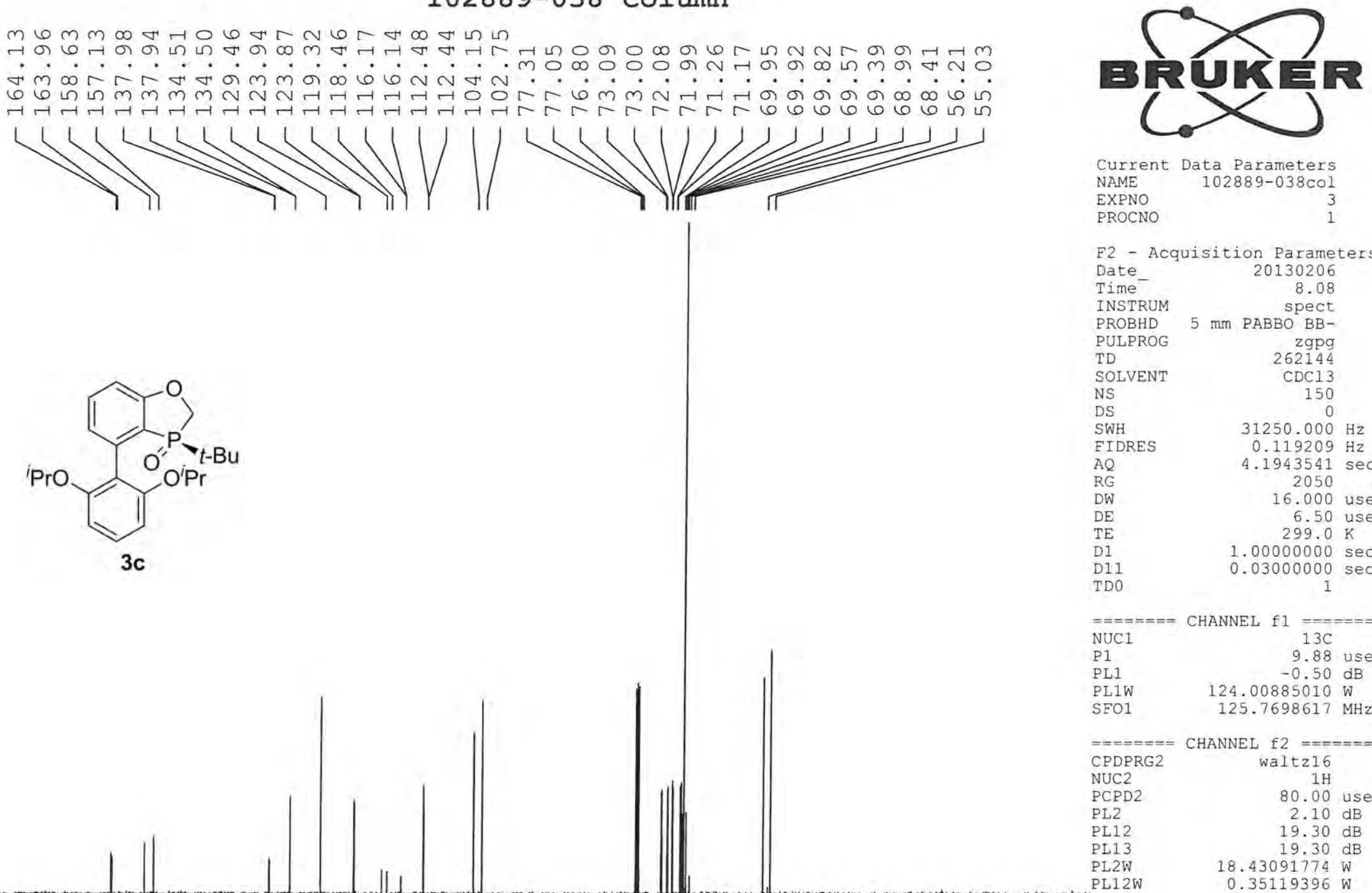

Current Data Parameters NAME $102889-038 \mathrm{col}$ EXPNO PROCNO

F2 - Acquisition Parameters Date_ 20130206 Time 8.08 SPect PROBHD 5 mI PABBO BBzgpg TD

SOLVENT

SWH $\quad 31250.000 \mathrm{~Hz}$ $0.119209 \mathrm{~Hz}$

AQ

$\mathrm{DW}$
$\mathrm{DE}$
$\mathrm{TE}$

TE

D11

TDO $.1943541 \mathrm{sec}$ 16.000 usec $299.0 \mathrm{~K}$

$1.00000000 \mathrm{sec}$

$=======$ CHANNEL $\mathrm{fl}$ NUC1

PL1

PLIW $13 \mathrm{C}$

SEO1

$=======$

CPDPRG

NUC2 2

PCPD2

PL2 2

PL12

PL2W

PL12W

SFO2

9.88 usec
$-0.50 \mathrm{~dB}$

$124.00885010 \mathrm{~W}$

$125.7698617 \mathrm{MHz}$

$500.1325007 \mathrm{MHz}$

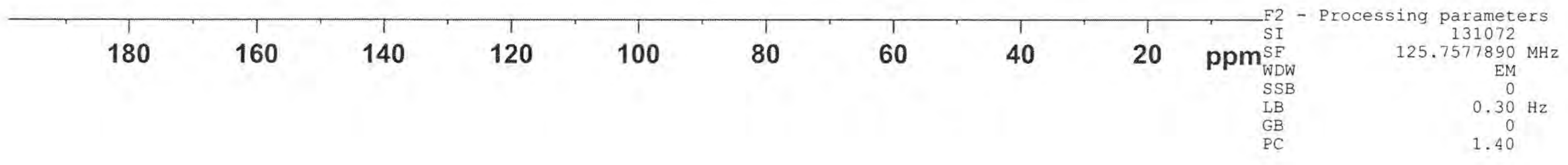




\section{9-094 column}

๘

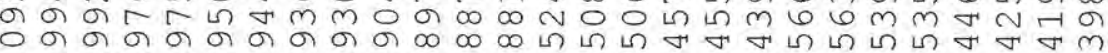

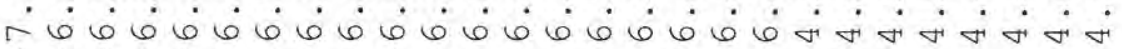

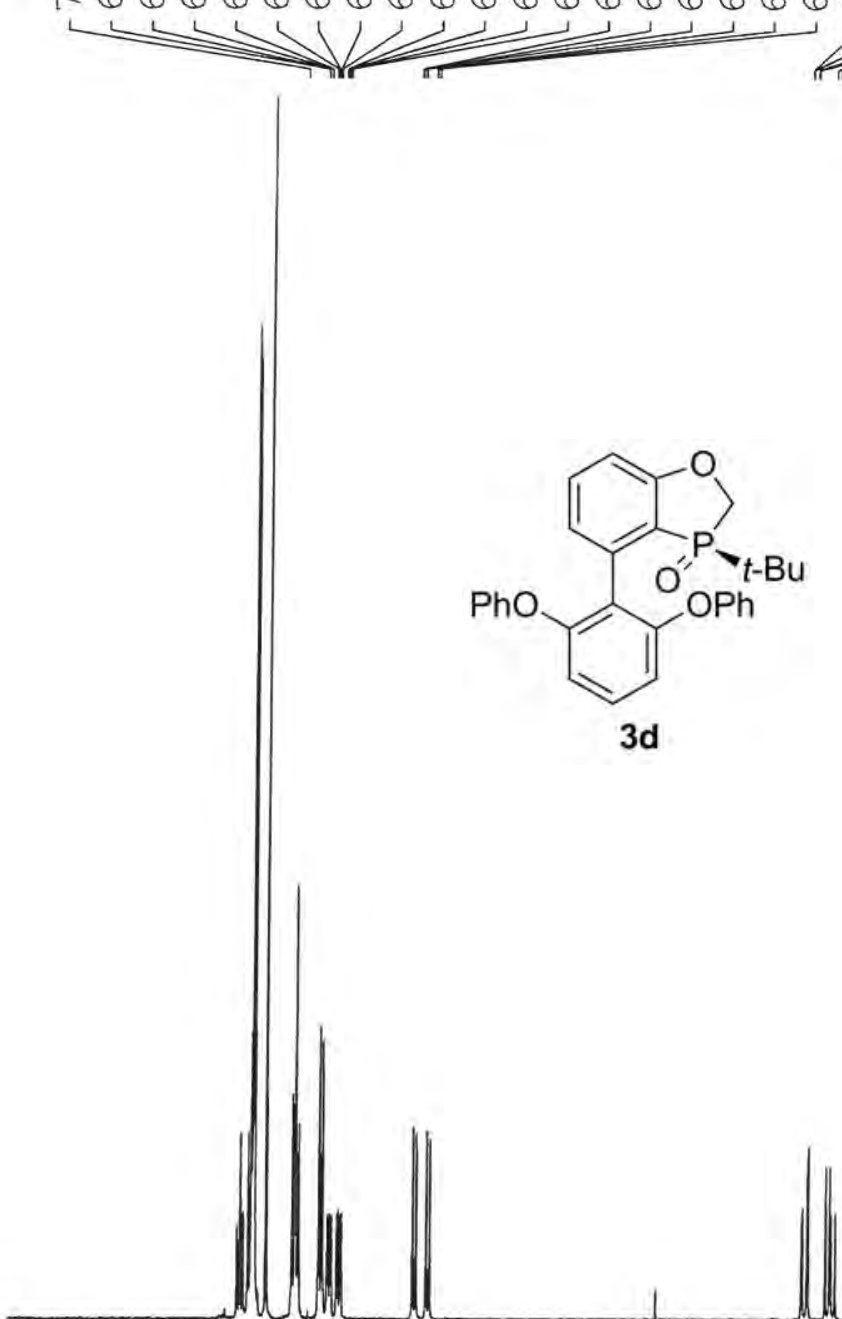

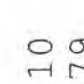

$\begin{array}{lll}-1 & 0 \\ -1 & 0\end{array}$

苑

$\sqrt{3}$

Current Data Parameters NAME

EXPNO

PROCNO

F2 - Acquisition Parameters

Date

20150717

Time- 15.09

INSTRUM

PROBHD

PULPROG

SOLVENT

NS

DS

SWH

FIDRES

$A Q$
$R G$
$D W$

DW

TE

D1

TDO

mm PABBO BB-

NUC1

P1

PL1

PLIW

q 2 2910

CDC13

16

$9014.423 \mathrm{~Hz}$

$0.275098 \mathrm{~Hz}$

$1.8175317 \mathrm{sec}$

512

55.467 usec

6.50 usec $299.0 \mathrm{~K}$

$2.00000000 \mathrm{sec}$

1

$==$ CHANNEL $\mathrm{f}$ $====$

1.95 usec $2.10 \mathrm{~dB}$ $18.43091774 \mathrm{~W}$

F2 - Processing parameters $\begin{array}{lc}\text { SI } & 16384 \\ \text { SE } & 500.1300138 \mathrm{MHz}\end{array}$ WDW

SSB
LB

EM

EM

PC 1.00

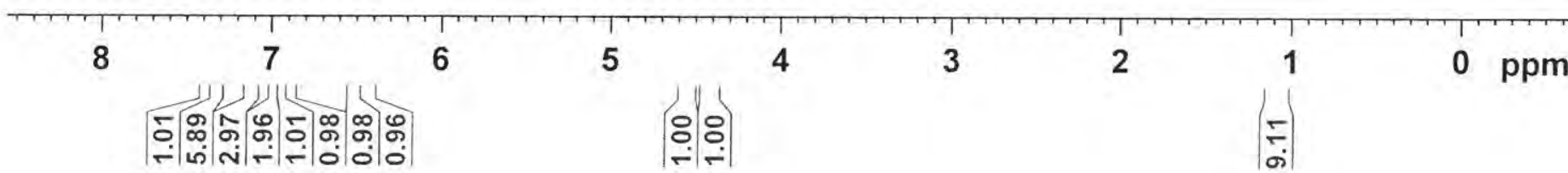




\section{9-094 column}

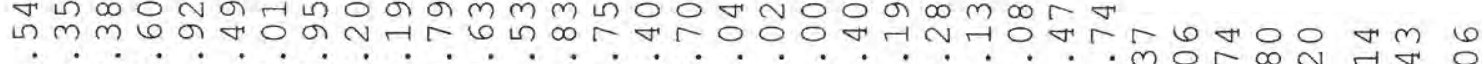
ก 6

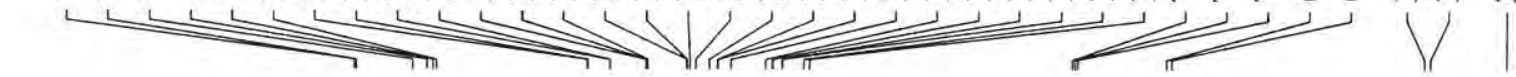

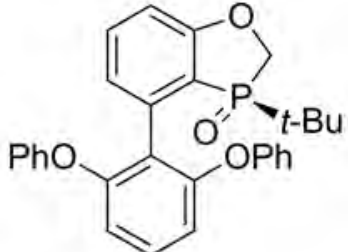

3d

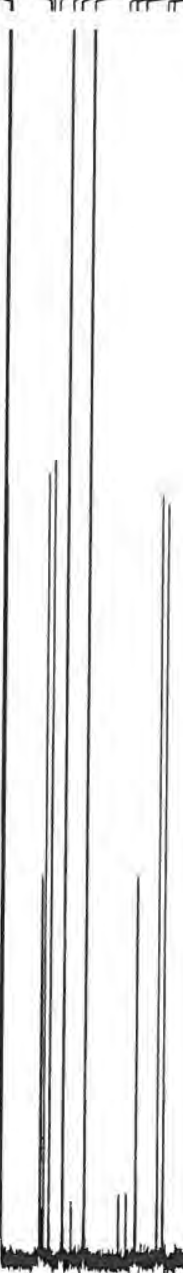

Current Data Parameters $103569-094$ EXPNO

$$
1
$$

F2 - Acquisition Parameters

Date_ 20151002

$\begin{array}{ll}\text { Time } & 17.57\end{array}$

spect
PROBHD $5 \mathrm{~mm}$ PABBO BB-

SOLVENT

NS

DS

SWH

FIDRES

$\mathrm{AQ}$
$\mathrm{RG}$

RG

$\mathrm{DE}$

TE

D1
d11
DELTA

TDO

SFO1

NUC1

P1

PLW1

$\mathrm{SFO} 2$

CPDPRG [2

PCPD2

PLW2

PLW12

zgpg
32768

$\mathrm{CDCl} 3$
300

$24038.461 \mathrm{~Hz}$

$24038.461 \mathrm{~Hz}$
$0.733596 \mathrm{~Hz}$

$0.6815744 \mathrm{sec}$ 2050

20.800 usec 6.50 usec $2.00000000 \mathrm{~K}$ $0.03000000 \mathrm{sec}$ $1.89999998 \mathrm{sec}$ 100.6243395 10.10 usec $-1.00000000 \mathrm{~W}$ $400.1316005 \mathrm{MH}$

$1 \mathrm{H}$

$$
\begin{array}{r}
1 \mathrm{H} \\
\text { waltzz } 16
\end{array}
$$$$
90.00 \text { usec }
$$

$-1.00000000 \mathrm{~W}$

$-1.00000000 \mathrm{~W}$

$-1.00000000 \mathrm{~W}$

F2 - Processing parameters

$\begin{array}{lc}\text { SI } & 65536 \\ \text { SE } & 100.6127690 \mathrm{MHz}\end{array}$

WDW $\quad 100.6127690$

SSB $\quad 0$

$1.00 \mathrm{~Hz}$

1. 40 


\section{9-056 column}

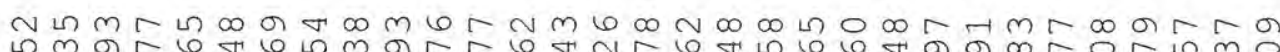

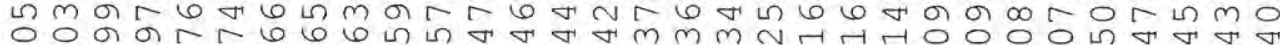

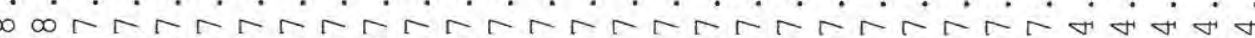

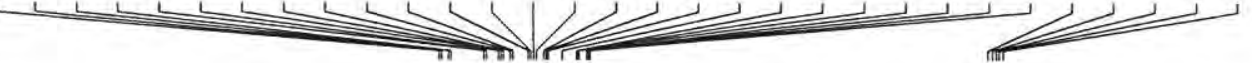

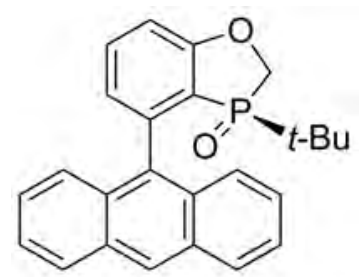

$3 e$

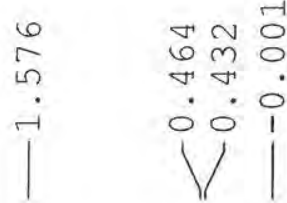

Current Data Parameters NAME $103669-056$ PROCNO

F2 - Acquisition Parameters

Date_ 20151009

Time ${ }^{-} \quad 15.39$

INSTRUM spect

PROBHD $5 \mathrm{~mm}$ PABBI $1 \mathrm{H}-$

TD

SOLVENT

NS

DS

SWH

FIDRES

$A Q$

RG

DW

$\mathrm{DE}$

D1

TDO

$q_{32} z 10$

$\mathrm{CDCl} 3$

16
0

$9014.423 \mathrm{~Hz}$

$0.275098 \mathrm{~Hz}$

$1.8175317 \mathrm{sec}$

1440

55.467 usec 6.50 usec $2.00000000 \mathrm{sec}$

$=== \pm== \pm=$ CHANNEL $\mathrm{fl}=======$ NUC1

P1

PL1

SFO1

$$
1 \mathrm{H}
$$

8.00 usec

14.98121262 W

$500.1334009 \mathrm{MH}$

F2 - Processing parameters

SI 16384

SF $\quad 500.1300144 \mathrm{MHz}$

EM

$\begin{array}{ll}\text { LB } & 0.30 \mathrm{~Hz}\end{array}$

$\begin{array}{ll}\mathrm{PB} & 1.00\end{array}$

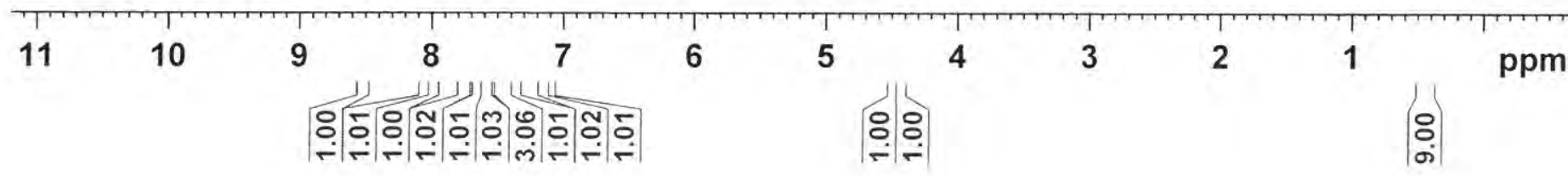




\section{9-056 column}

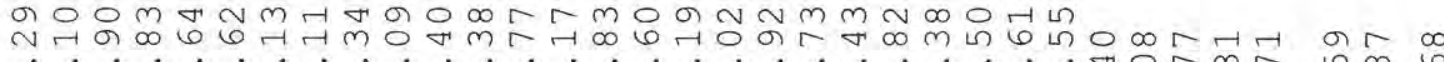

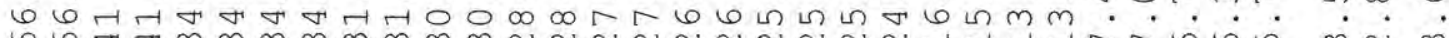

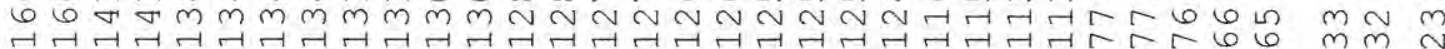

$\underbrace{2,1}_{1}$

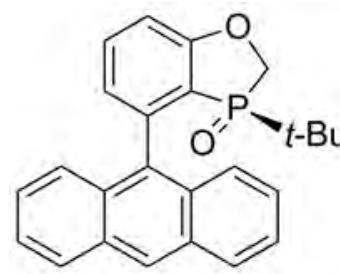

3e
Current Data Parameters NAME $103669-056$ EXPNO

F2 - Acquisition Parameters

Dater 20151009

Time $^{-} \quad 16.20$

spect

PROBHD $5 \mathrm{~mm}$ PABBO BB-

PULPROG

TD

SOLVENT

NS

SWH

FIDRES

AQ

RG

RW

DE

TE

d11

DELTA

TDO

SEO1

NUC1

P1

PLW1

$\mathrm{SFO} 2$
$\mathrm{NUC2}$

CPDPRG [2

PCPD2

PIW2

PI 2

E2 - Processing parameter

$\begin{array}{lr}\text { SI } & 65536 \\ \text { SF } & 100.6127690 \mathrm{MHz}\end{array}$

SSB $\quad 0$
(ISB
$1.00 \mathrm{~Hz}$ 
$\bullet \forall \odot m の \sim N \cdots$

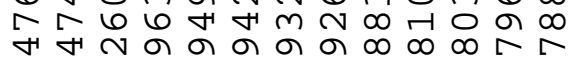
ن

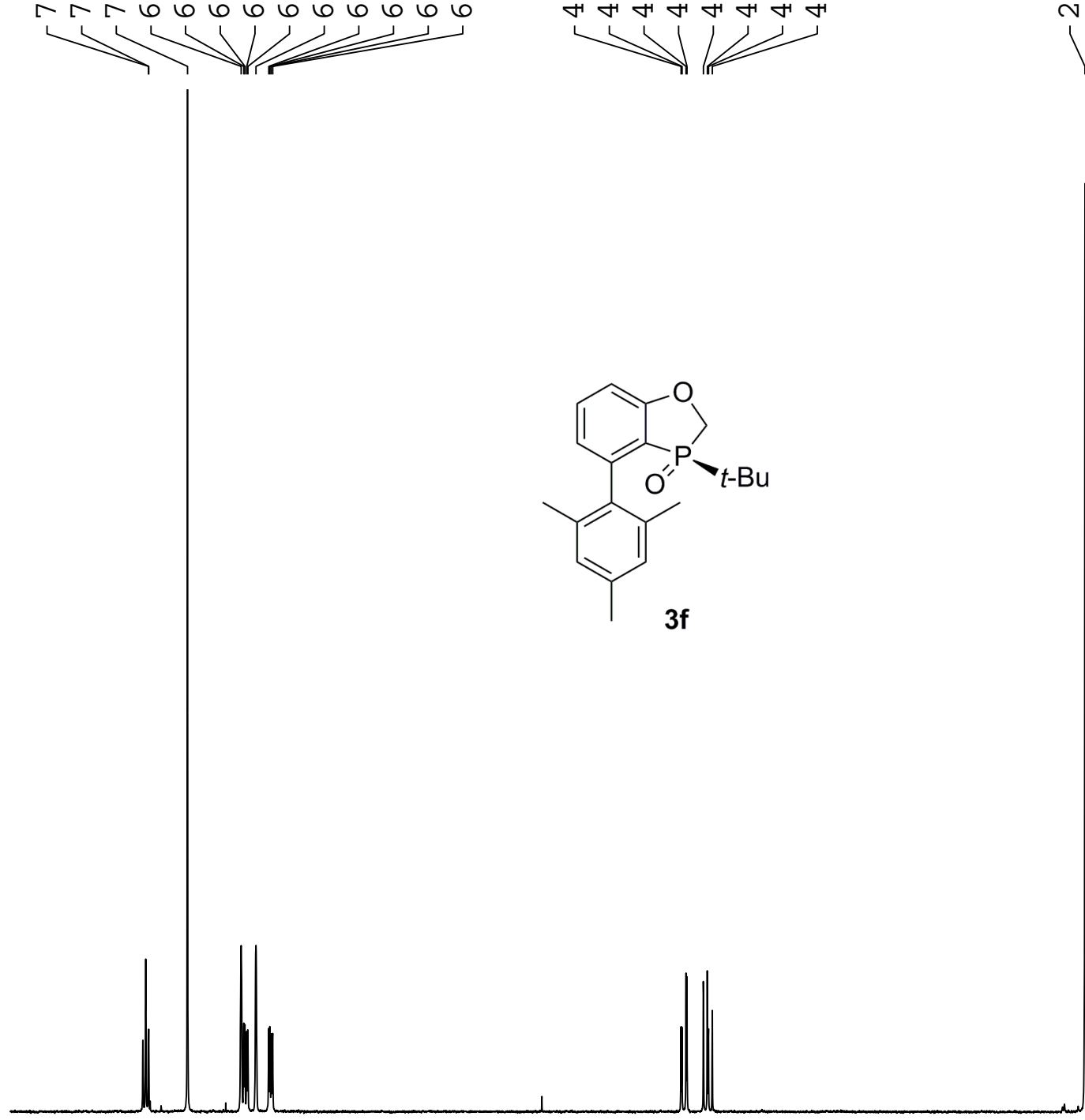

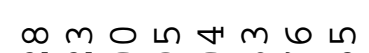

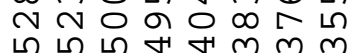

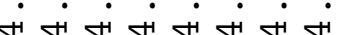
농 N ก

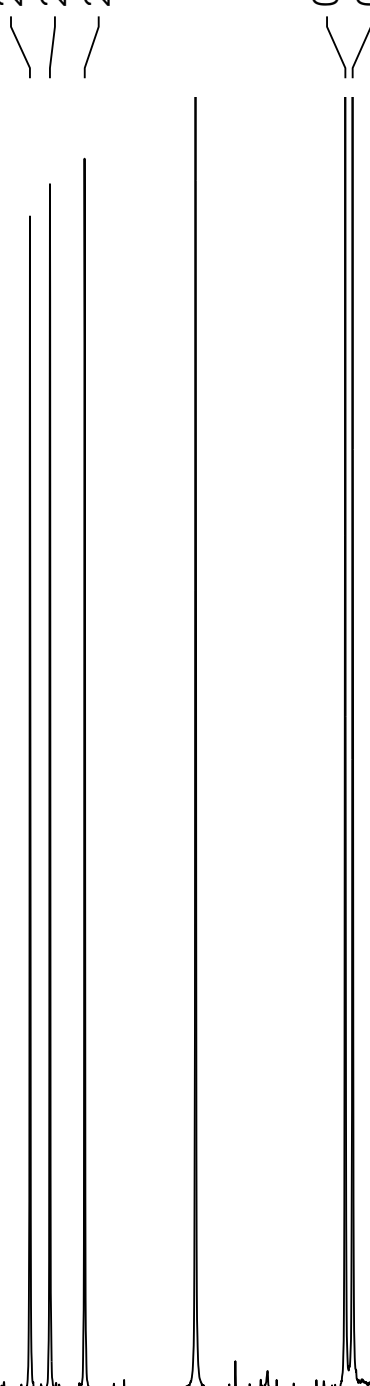

10
20
5
0
0
0
0
R

Current Data Parameters NAME

EXPNO 103669-090 PROCNO

F2 - Acquisition Parameters Date_ 20151105 $\begin{array}{ll}\text { Time } & 10.37\end{array}$ PROBHD $5 \mathrm{~mm}$ PABBI $1 \mathrm{H}-$ PULPROG 5 mm PABBI 1 TD SOLVENT CDCl3 NS DS SWH FIDRES $\quad 9014.423 \mathrm{~Hz}$ $0.275098 \mathrm{~Hz}$ RG DW DE TE TDO $2.00000000 \mathrm{Kec}$ 1

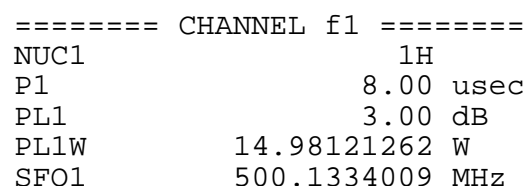

F2 - Processing parameters $\begin{array}{lr}\text { SI } & 16384 \\ \text { SF } & 500.1300137 \\ \text { MHz }\end{array}$ WDW $\begin{array}{lll}\text { SSB } & 0 & 0.30 \mathrm{~Hz}\end{array}$ GB $\mathrm{PC}$

1.00

\section{$\begin{array}{lllllllllllllllll}8.0 & 7.5 & 7.0 & 6.5 & 6.0 & 5.5 & 5.0 & 4.5 & 4.0 & 3.5 & 3.0 & 2.5 & 2.0 & 1.5 & 1.0 & 0.5 & \text { ppm }\end{array}$

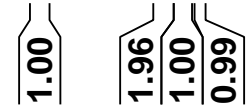 $\left|\begin{array}{l}\infty \\ 0 \\ 0 \\ 0\end{array}\right| \begin{aligned} & \infty \\ & 0 \\ & 0\end{aligned} \mid$




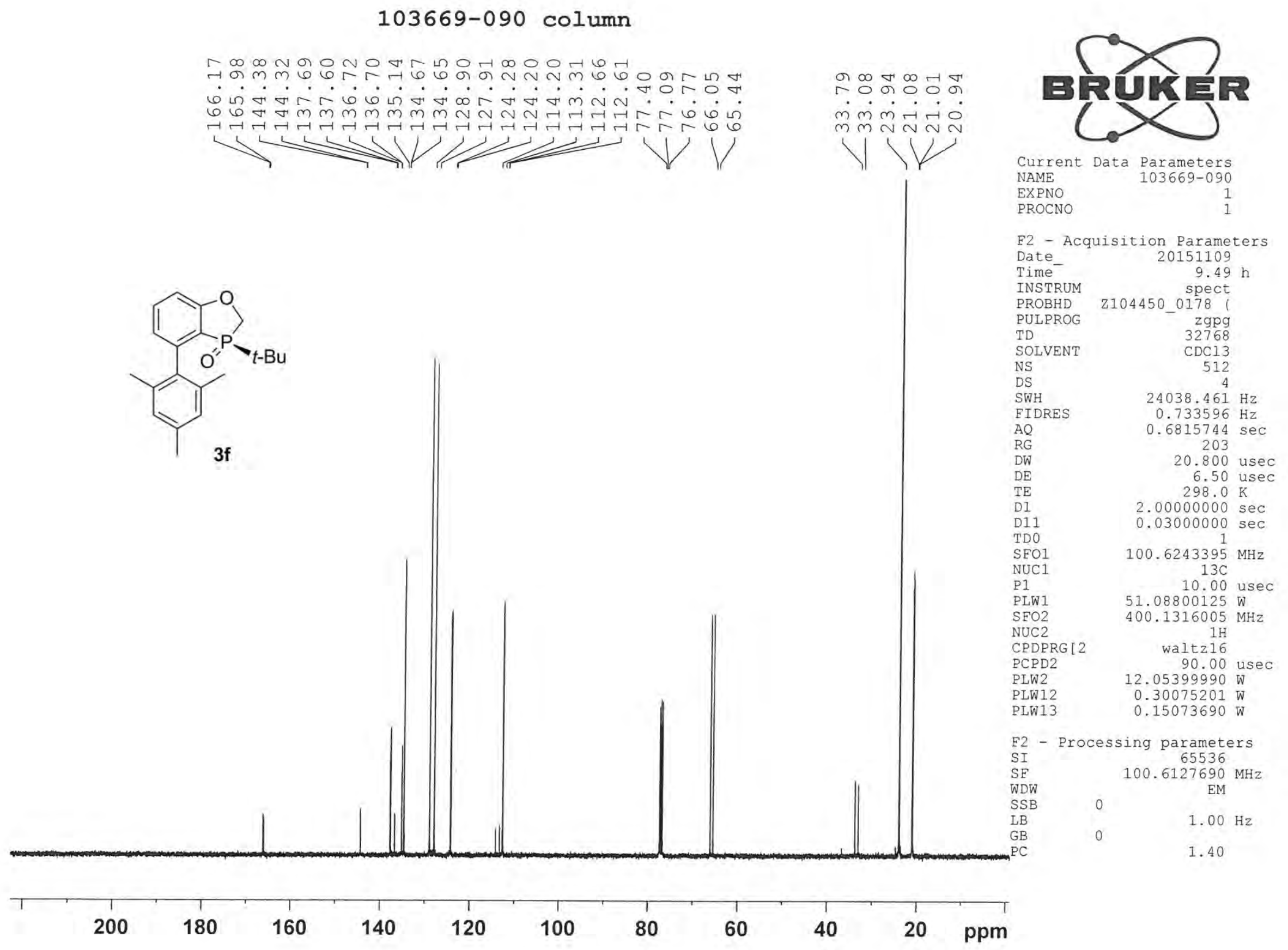




\section{9-058 column}

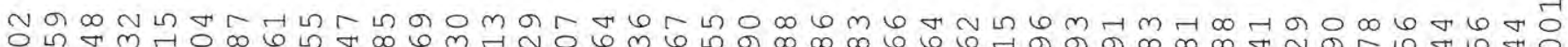

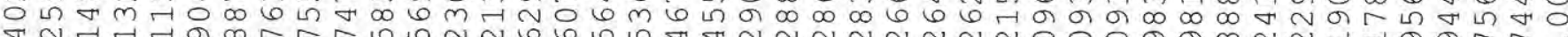
rír1

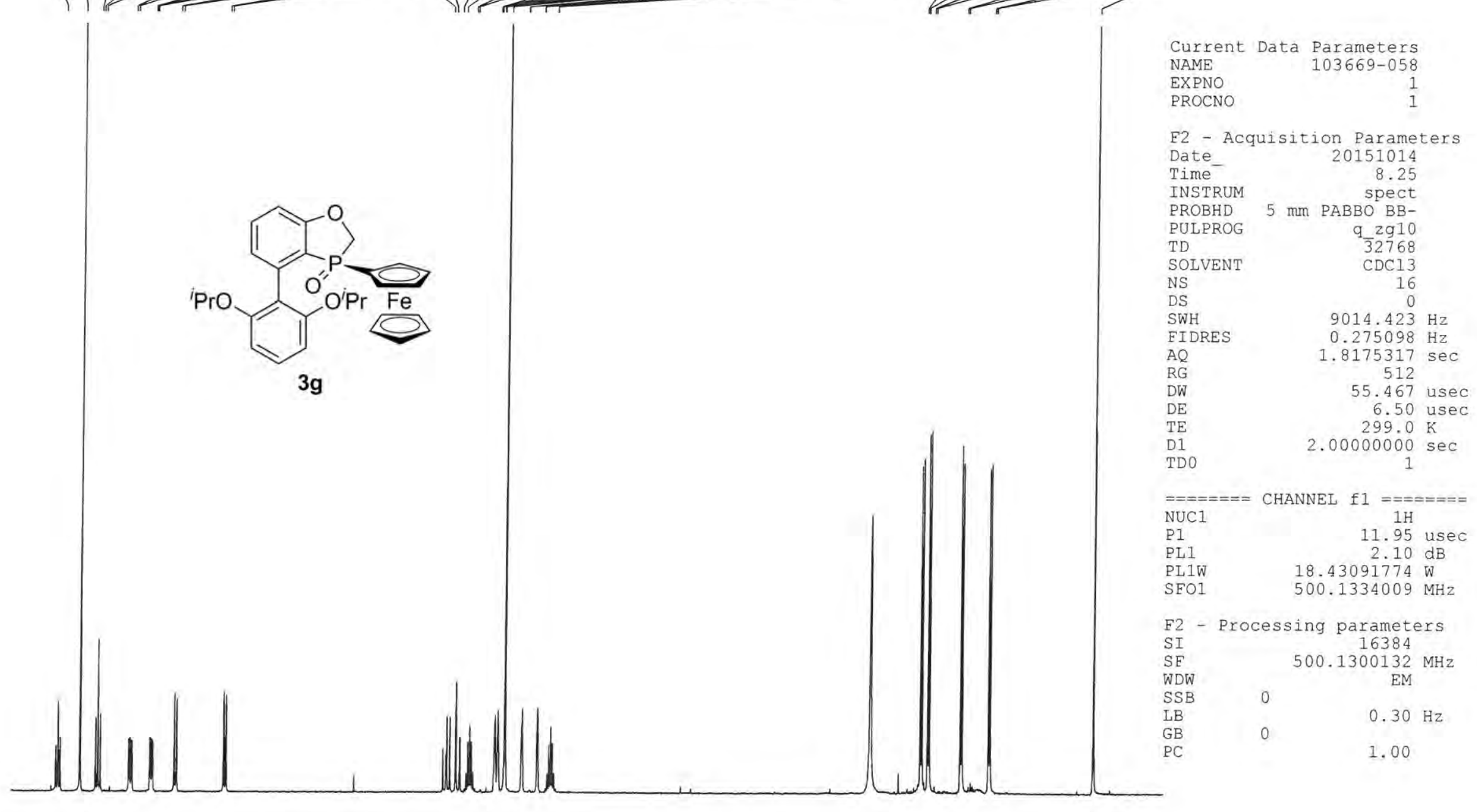

7

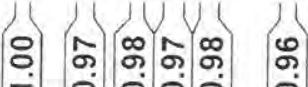
o

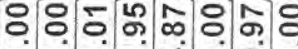

6
5
3
2
1

ริ ภิ สิ

लें लं| ppm 


\section{9-058 column}

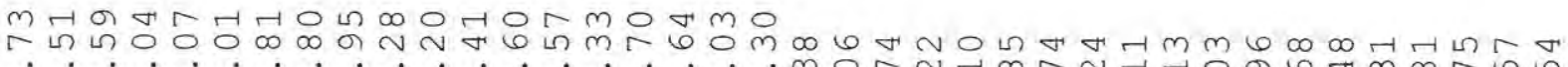

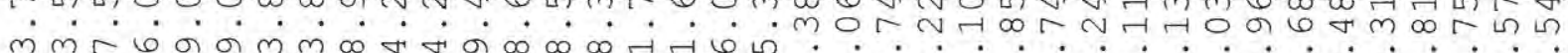

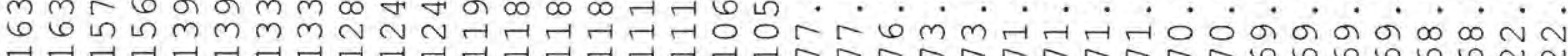

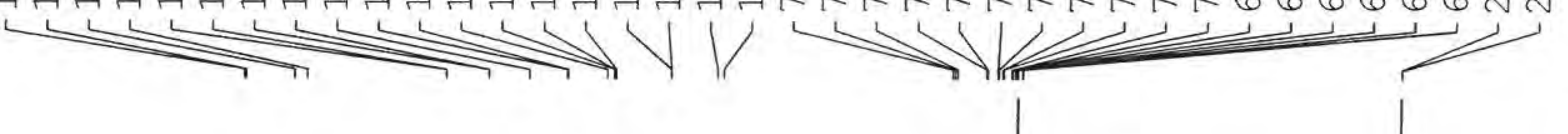

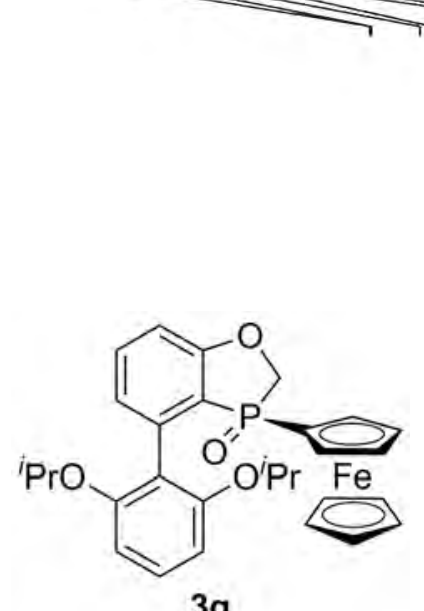

$3 \mathrm{~g}$

Current Data Parameters $103669-058$ EXPNO

F2 - Acquisition Parameters

Date_ 20151014

Time 16.24

INSTRUM SPect

PROBHD $5 \mathrm{~mm}$ PABBO BB-

TD

SOLVENT

NS

DS

SWH

FIDRES

NUC2

CPDPRG [2

PCPD2

PLW2

PLW12

PLW13

$$
\begin{array}{r}
\text { zgpg } \\
32768 \\
\text { CDC13 } \\
256 \\
4 \\
24038.461 \mathrm{~Hz} \\
0.733596 \mathrm{~Hz} \\
0.6815744 \mathrm{sec} \\
2050 \mathrm{usec} \\
20.800 \mathrm{usec} \\
6.50 \mathrm{usec} \\
298.0 \mathrm{~K} \\
2.0000000 \mathrm{sec} \\
0.03000000 \mathrm{sec} \\
1.89999998 \mathrm{sec} \\
1 \\
100.6243395 \mathrm{MHz} \\
13 \mathrm{C} \\
10.10 \mathrm{usec} \\
-1.00000000 \mathrm{~W} \\
400.1316005 \mathrm{MHz} \\
1 \mathrm{H} \\
\text { waltz16 } \\
90.00 \mathrm{usec} \\
-1.00000000 \mathrm{~W} \\
-1.00000000 \mathrm{~W} \\
-1.00000000 \mathrm{~W}
\end{array}
$$

F2 - Processing parameters SI $\quad 65536$ SF $\quad 100.6127690 \mathrm{MH}$ EM

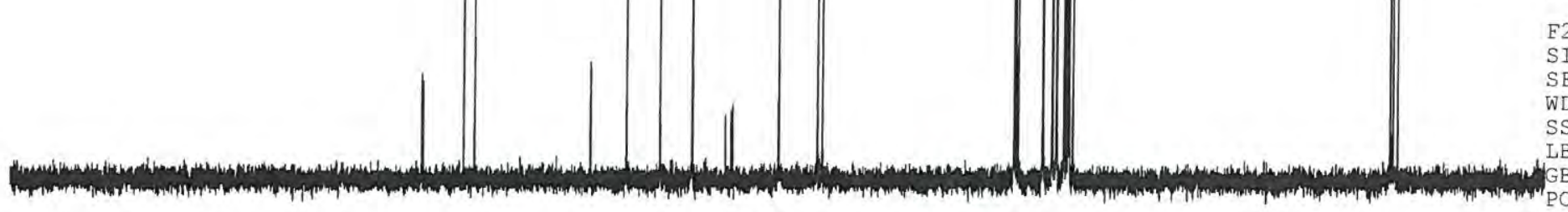

$1.00 \mathrm{~Hz}$ 


\section{9-068 column}

m ○ढ̆

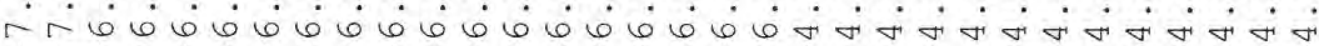

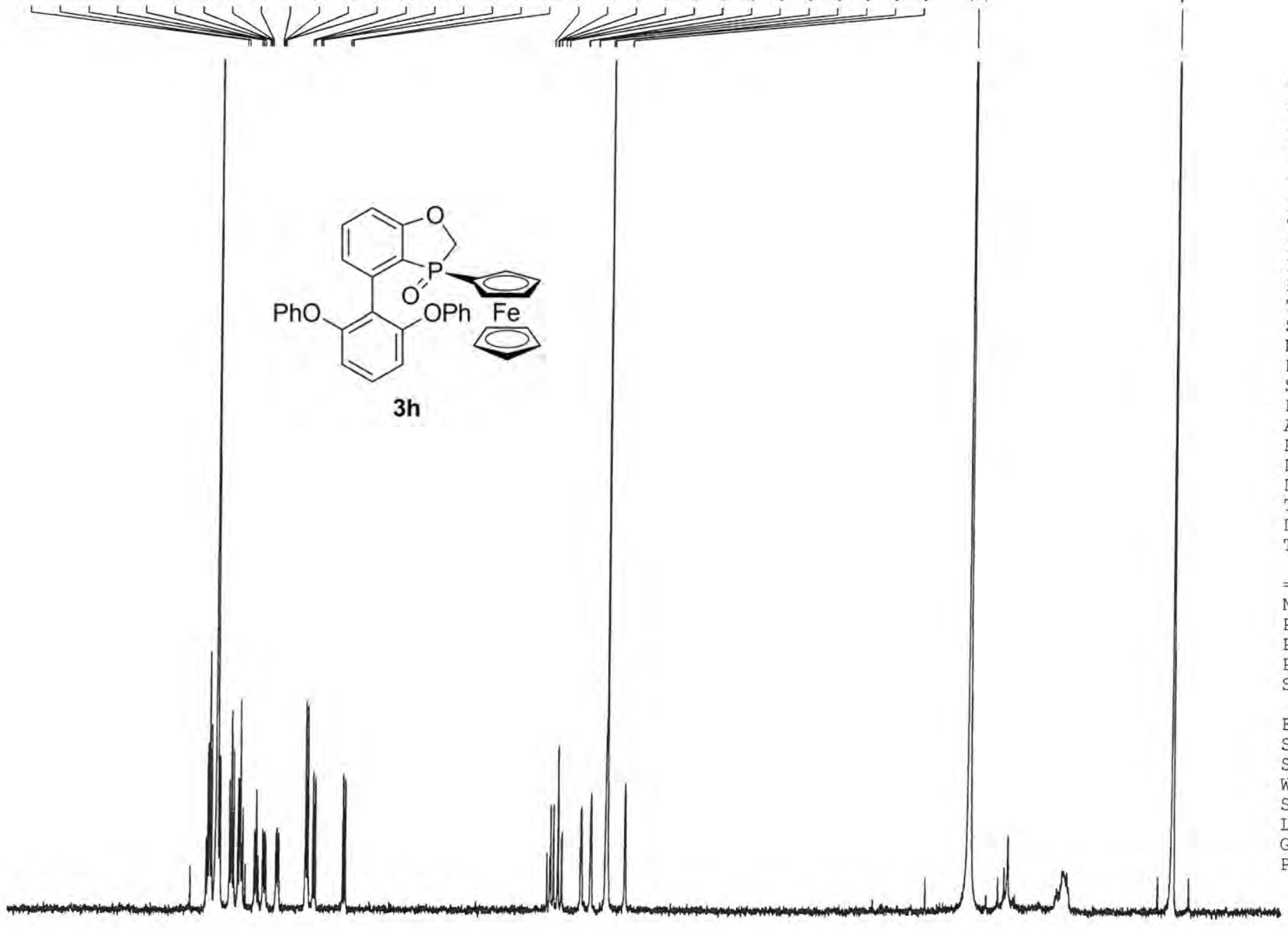

Current Data Parameter

E2 - Acquisition Parameters

Date 20151023

Time

15.30

INSTRUM

PROBHD

PULPROG

$5 \mathrm{~mm}$ PABBI $1 \mathrm{H}-$

SOLVENT

NS

SWH

FIDRES

AQ
RG
DW

$D E$

TE

TDO

q $\mathrm{zg} 10$

$\mathrm{CDCl}$

0
0
$9014.423 \mathrm{~Hz}$

$0.275098 \mathrm{~Hz}$

$1.8175317 \mathrm{~s}$

55.467 usec

5.467 usec
6.50 usec $299.0 \mathrm{~K}$

$========$ CHANNEL $\mathrm{f} 1$

NUC

1

PL1W

PL1K

8.00 usec

14.98121262 W

2 - Processing parameters

SI 16384

WDW $\quad 500.1300139 \mathrm{MH}$

SSB $\quad 0$

LB $0.30 \mathrm{~Hz}$

$\begin{array}{lll}\text { GB } & 0 & 1.00\end{array}$

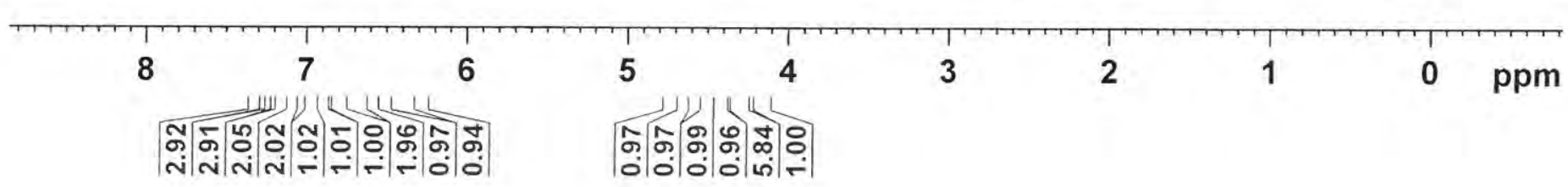




\section{9-068}

Hू,

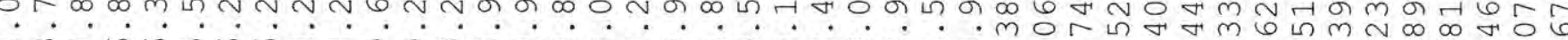

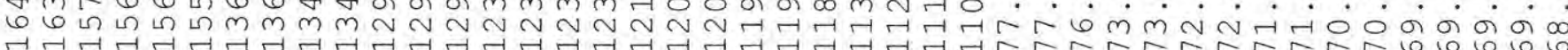

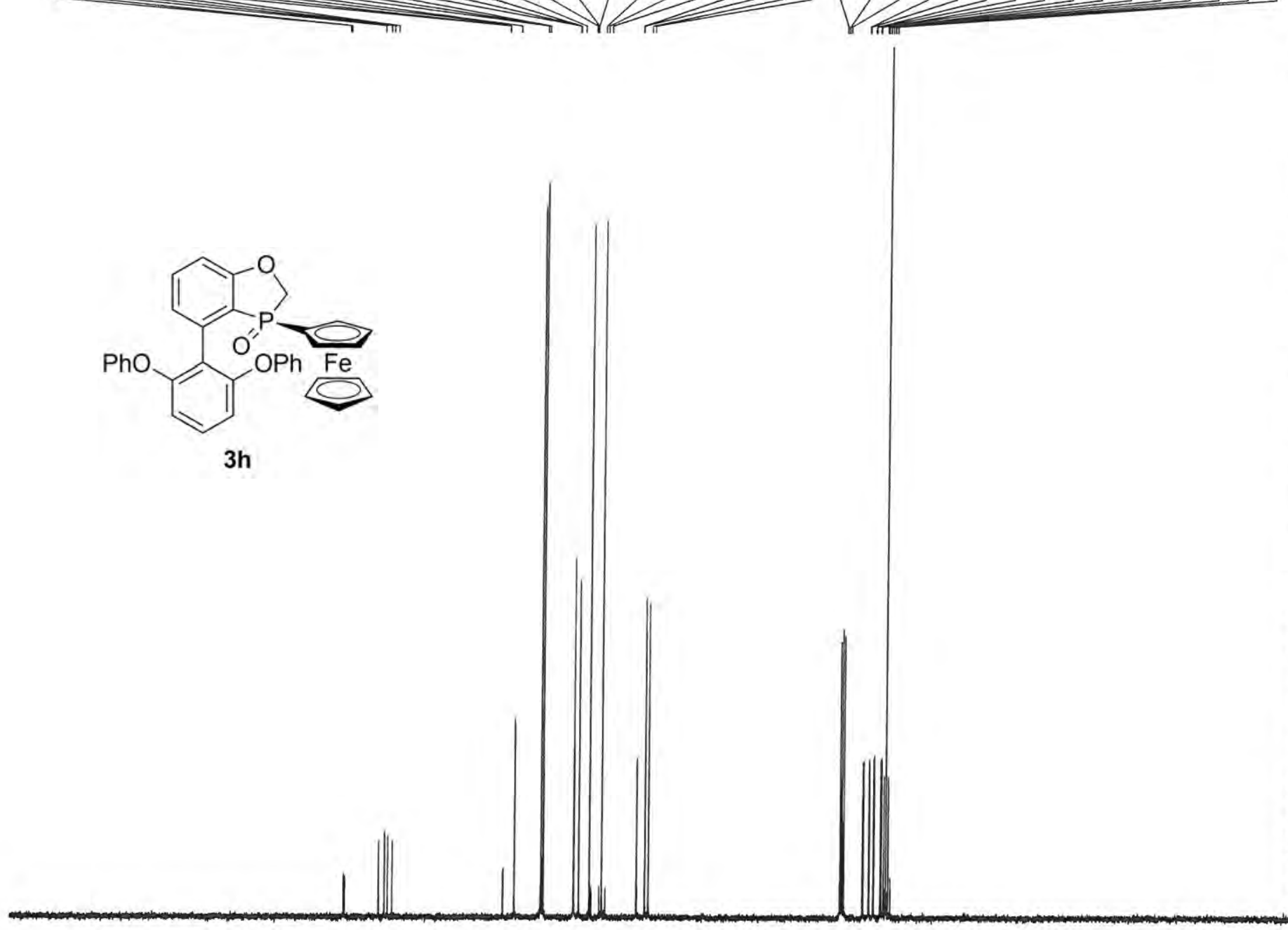

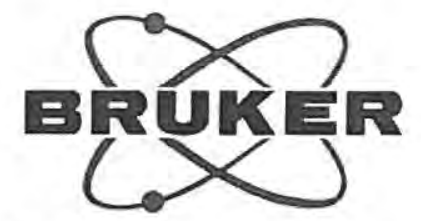

Current Data Parameters NAME PROCNO

F2 - Acquisition Parameters

Date_ 20151028

Time $18.43 \mathrm{~h}$

INSTRUM

PROBHD 2104450_0178

PULPROG
zgpg

TD 32768

NS

SWH

$24038.461 \mathrm{~Hz}$

$0.733596 \mathrm{~Hz}$

RG

DW

$\mathrm{DE}$

TE

D11

TDO

SEO1

PLW1
SFO2
NUC2

NUC2 2

CPDPRG [

PCPD2

10

PLW12

$.6815744 \mathrm{sec}$

203

20.800 usec 6.50 use

$2.00000000 \mathrm{sec}$ 0.03000000 sec 1 $100.6243395 \mathrm{MHz}$ $13 \mathrm{C}$

10.00 use $400.1316005 \mathrm{~W}$

$$
\text { waltz } 16
$$

90.00 usec

$12.05399990 \mathrm{~W}$

$0.30075201 \mathrm{~W}$

$0.15073690 \mathrm{~W}$

F2 - Processing parameters

SI Processing parameter

SF $\quad 100.6127690 \mathrm{MH}$

SSB 0

$100.6127690 \mathrm{MH}$

$1.00 \mathrm{~Hz}$

1.40 


\section{9-064 column}

가구

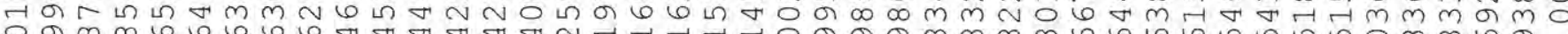

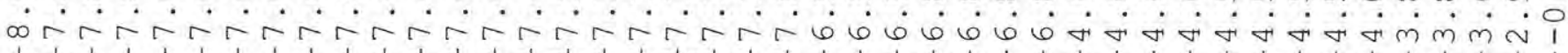

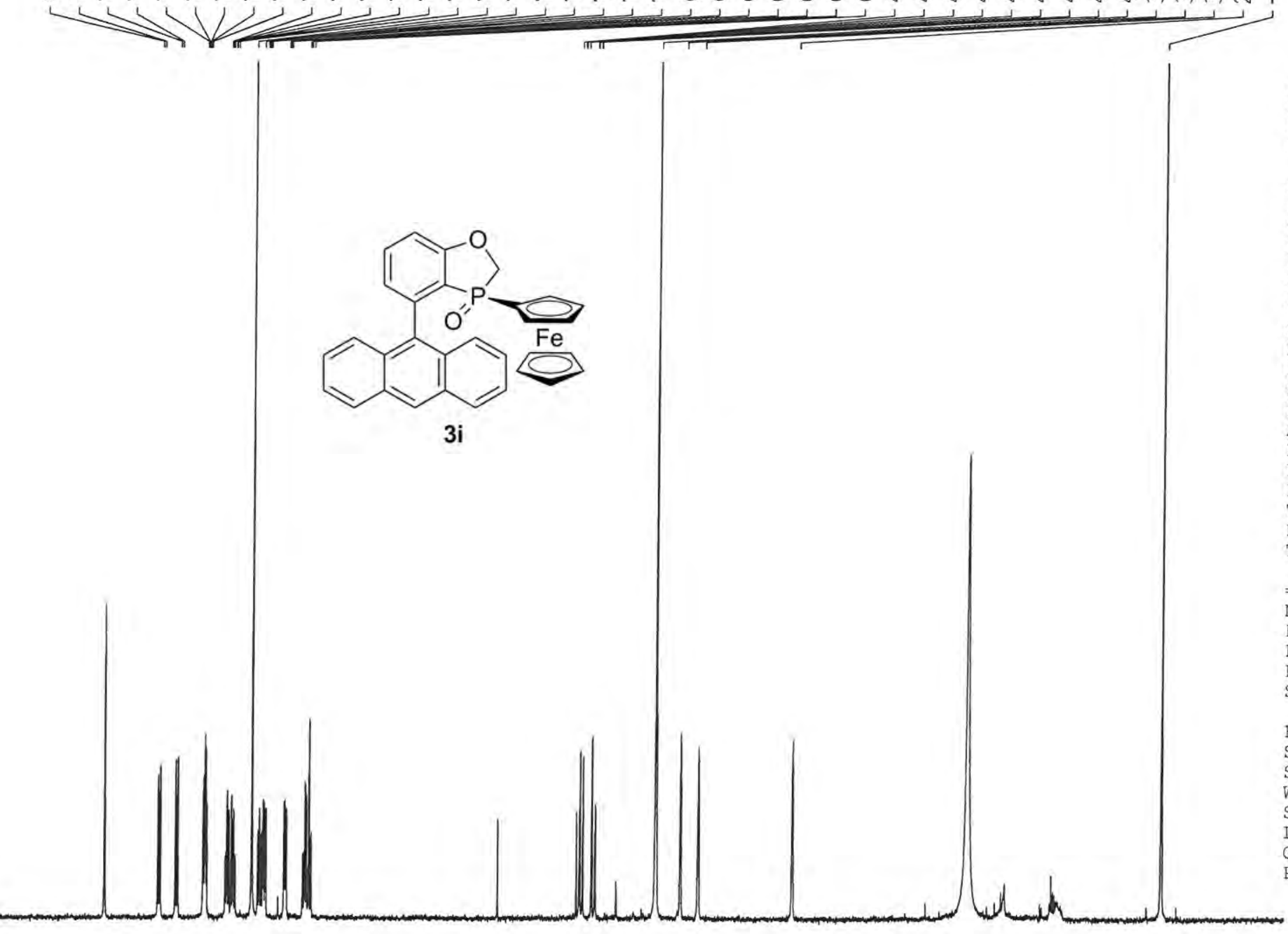

Current Data Parameters NAME 103669-06 PROCNO

E2 - Acquisition Parameters

Date 20151023

$\begin{array}{lr}\text { Time } & 2013.02 \\ & \end{array}$

INSTRUM spect

PULPROG $5 \mathrm{~mm}$ PABBI 1 I

TD $\overline{3} 2768$

SOLVENT CDC13

SWH

FIDRES $\quad 9014.423 \mathrm{~Hz}$

$0.275098 \mathrm{~Hz}$

AQ

DW

$\mathrm{DE}$

TE

TDO

$1.8175317 \mathrm{sec}$

55.467 usec

6.467 usec

2.50 usec

$========$ CHANNEI $\mathrm{f}$

NUC1

P1 1

3.00 dB

SFO1 $\quad \begin{array}{ll}14.98121262 \\ \text { W }\end{array}$

F2 - Processing parameters

SI 16384

$\begin{array}{lr}\text { SE } & 500.1300140 \mathrm{MHz} \\ \text { WDW } & \text { EM }\end{array}$

$\begin{array}{lll} & & 0.30 \mathrm{~Hz}\end{array}$

1. 00

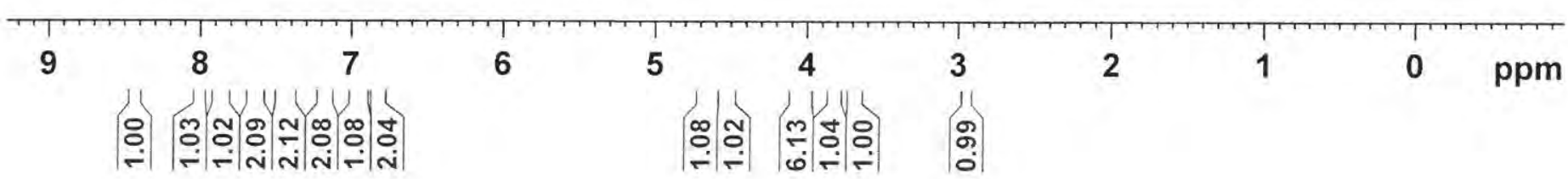




\section{9-064}

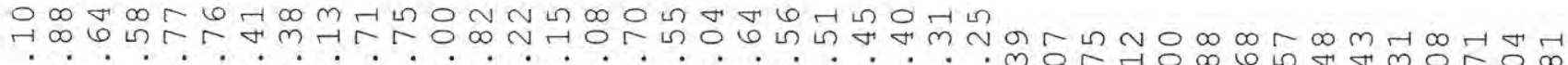

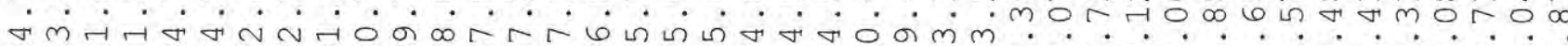

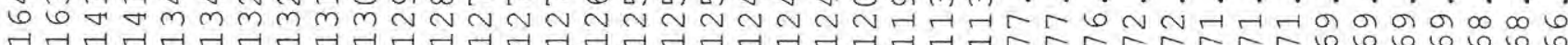

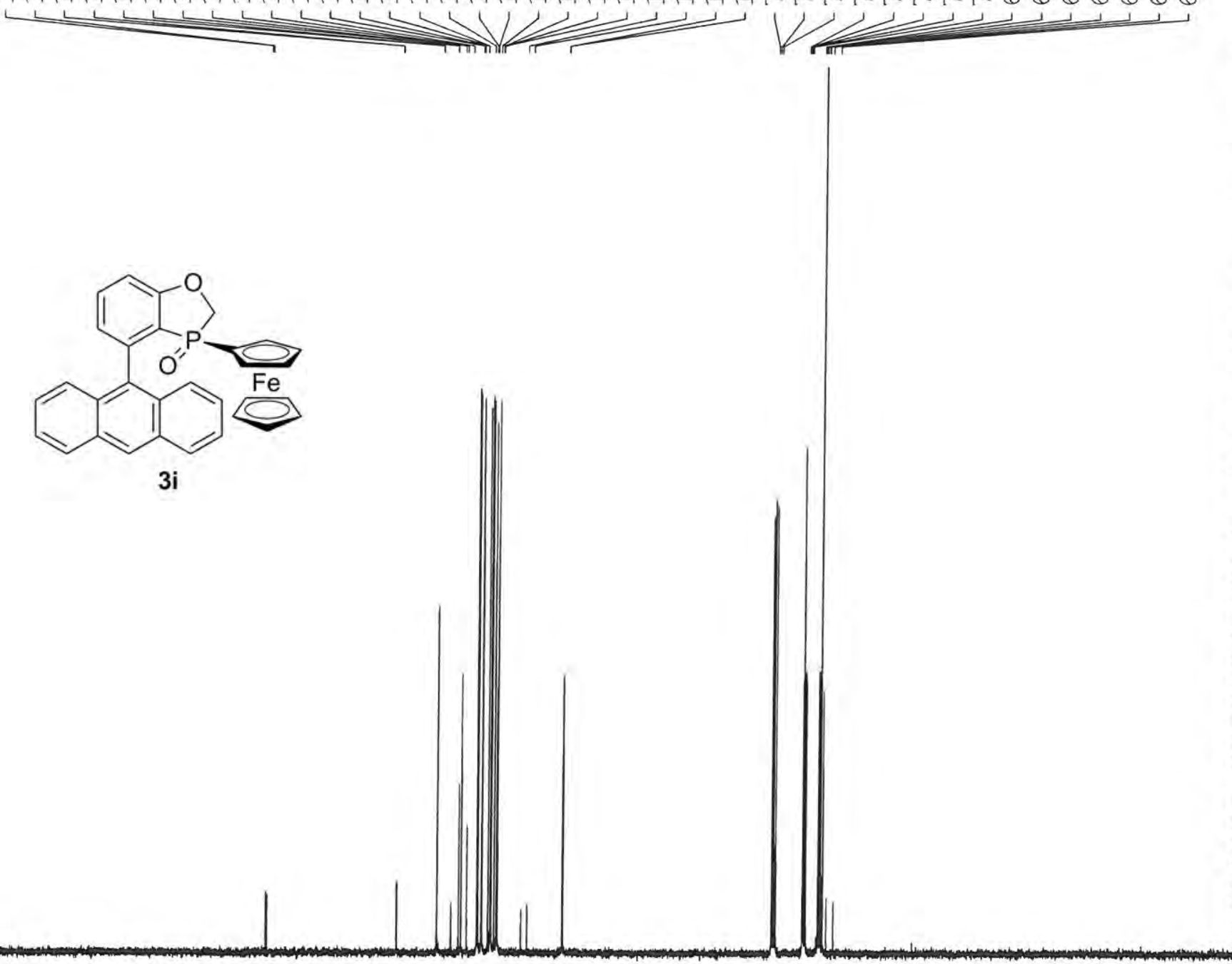

Current Data Parameters NAME 103669-064 EXPNO

F2 - Acquisition Parameters

Date_ 20151028

Time $17.06 \mathrm{~h}$

INSTRUM spect

PROBHD Z104450_0178 (

$\mathrm{zgpg}$
PULPROG
32768

$\begin{array}{ll}\text { TD } & 32768 \\ \text { SOLVENT } & \text { CDC13 }\end{array}$

NS $\quad 1024$

$\begin{array}{lr}\text { SWH } & 24038.461 \mathrm{~Hz} \\ \text { EIDRES } & 0.733596 \mathrm{~Hz}\end{array}$

$0.6815744 \mathrm{sec}$

RG

RG
DW
DE

$\mathrm{DE}$

D1

D11

TDO

NUC1

$\mathrm{P} 1$
$\mathrm{PLW} 1$
$\mathrm{SFO} 2$

$\mathrm{SFO} 2$

CPDPRG

PCPD2

(1)

PLW13

20.800 usec 6.50 usec
$298.1 \mathrm{~K}$

$2.00000000 \mathrm{sec}$ $0.03000000 \mathrm{sec}$ $100.6243395 \mathrm{MHz}$ $13 \mathrm{C}$
10.00 use $51.08800125 \mathrm{~W}$ $400.1316005 \mathrm{MHz}$

$1 \mathrm{H}$

$$
\text { waltz } 16
$$

90.00 use

$12.05399990 \mathrm{~W}$

$0.15073690 \mathrm{~W}$

F2 - Processing parameters SI 65536 100.6127690 MH SSB $\quad 0$

GB

$1.00 \mathrm{~Hz}$ 


\section{9-042 column}

NmGN 约 rarararar í

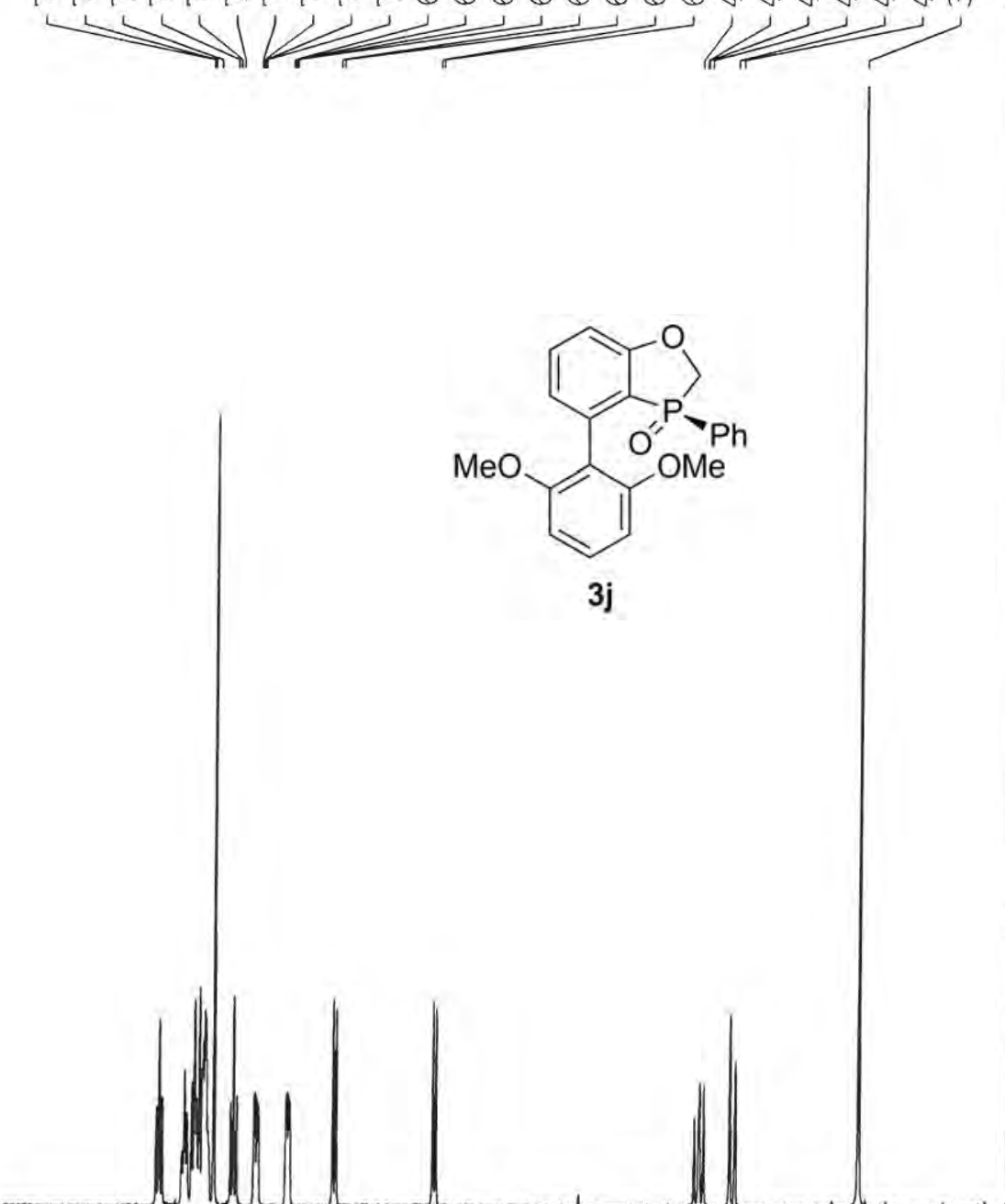

Urrent Data Parameters NAME $103669-042$ PROCNO

F2 - Acquisition Parameters

Date_ 20150930

$\begin{array}{lr}\text { Time } & \\ & \end{array}$

INSTRUM spect

PROBHD $5 \mathrm{~mm}$ PABBI $1 \mathrm{H}-$

PULPROG q zg10

TD

SOLVENT

NS

DS

SWH

EIDRES

RG

DW

$\mathrm{DE}$

TE

TDO

$\mathrm{CDC} 13$

16
0
$9014.423 \mathrm{~Hz}$
$0.275098 \mathrm{~Hz}$
$1.8175317 \mathrm{~Hz}$

$1.8175317 \mathrm{sec}$

2050
55.467 usec

6.50 usec

$299.0 \mathrm{~K}$

$===== \pm==$ CHANNEL $\mathrm{f}$

NUC1

P1

PI1

PL1 $3.00 \mathrm{~dB}$

$14.98121262 \mathrm{~W}$

F2 - Processing parameters

SI Processing pa

SI $\quad 500.1300139$

SE $\quad 500.1300139 \mathrm{MH}$

SSB $\quad 0 \quad$ EM

LB $\quad 0.30 \mathrm{~Hz}$

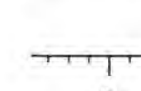

8 7

6

5

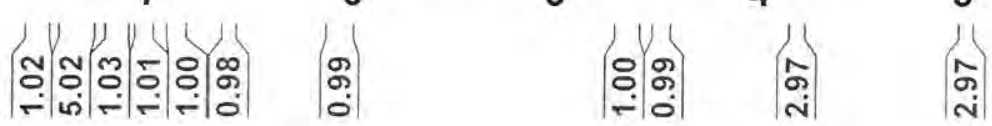

4

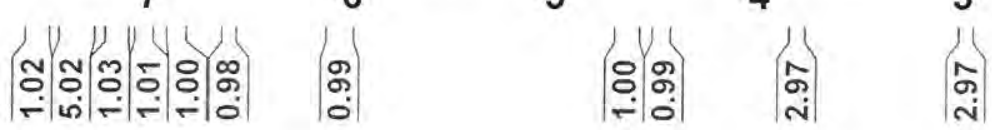

3

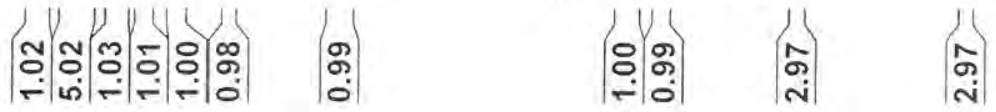




\section{9-042 column}

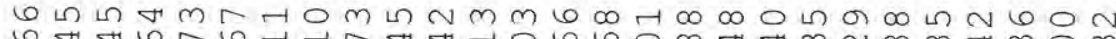

,

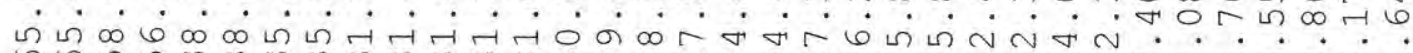

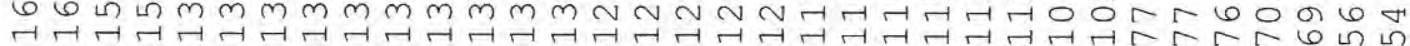

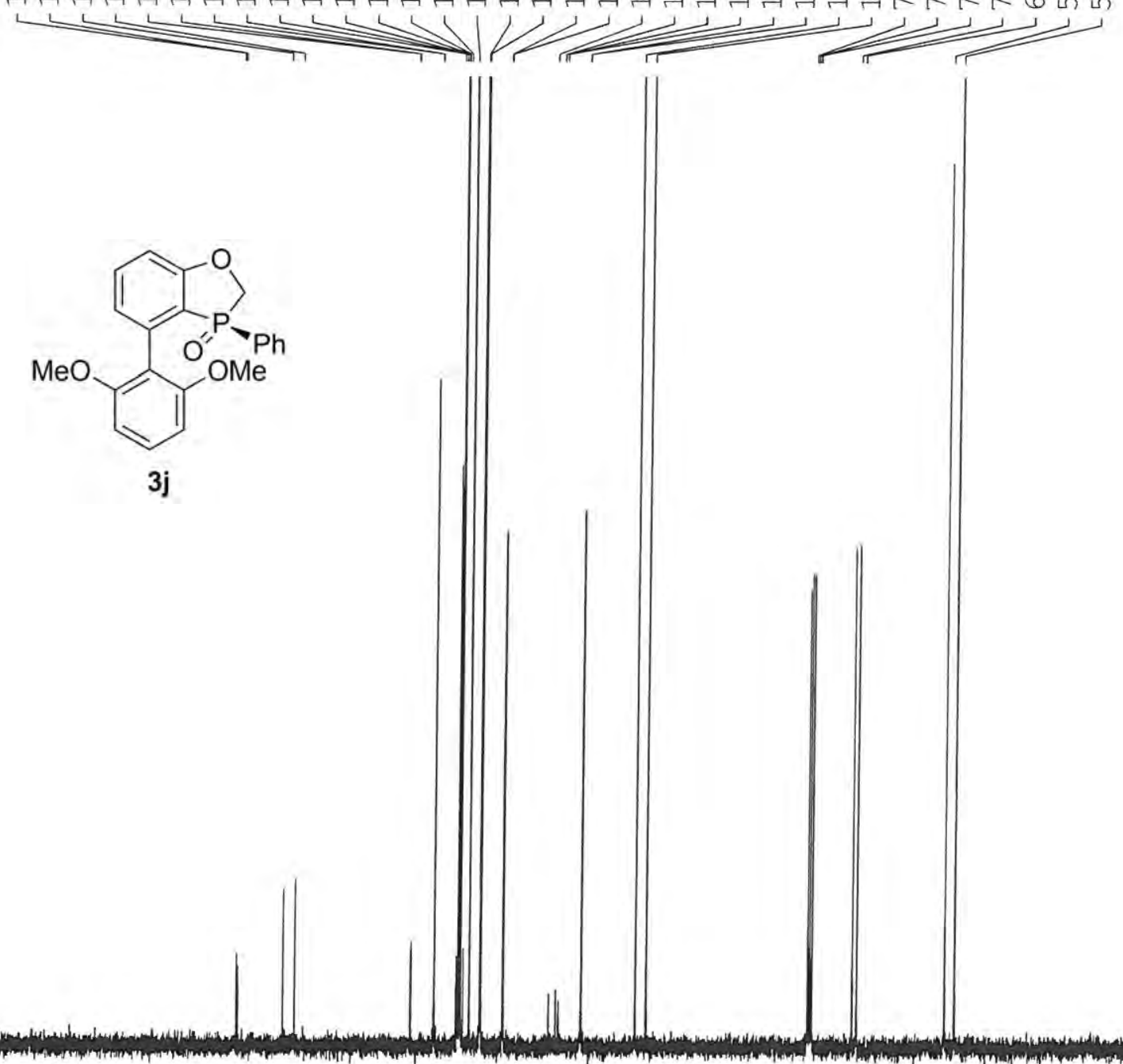

Current Data Parameters

NAME $103669-042$

EXPNO

1

F2 - Acquisition Parameters

Dater 20151002

Time- 16.11

INSTRUM 5 mect

PROBHD $5 \mathrm{~mm}$ PABBO BB-

TD

SOLVENT

NS

DS

SWH

FIDRES

$A Q$
$R G$

SFO1

(

PLW

SEO2

NUC2

CPDPRG [ 2

PCPD2

PIV2

PLW12

$$
\begin{array}{r}
\text { zgpg } \\
32768 \\
\text { CDC13 } \\
226 \\
4 \\
24038.461 \mathrm{~Hz} \\
0.733596 \mathrm{~Hz} \\
0.6815744 \mathrm{sec} \\
2050 \\
20.800 \text { usec } \\
6.50 \text { usec } \\
298.0 \mathrm{~K}
\end{array}
$$

$2.00000000 \mathrm{sec}$ $0.03000000 \mathrm{sec}$ 1.89999998 se $100.6243395 \mathrm{MH}$

10.10 usec $000000 \mathrm{~W}$ $400.1316005 \mathrm{MHz}$

$$
\text { waltz } 16
$$

waltzi6

$-1.00000000$

$-1.00000000 \mathrm{~W}$

$-1.00000000 \mathrm{~W}$

F2 - Processing parameters

$\begin{array}{ll}\text { SI } & 65536 \\ \text { SE } & 100.6127690 \mathrm{MHz}\end{array}$

SF

SSB

$100.6127690 \mathrm{MHz}$

$1.00 \mathrm{~Hz}$

1.40 


\section{9-044 column}

ㄴ. vo 0 。

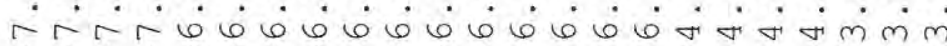

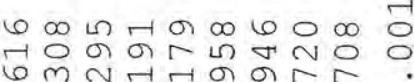
मित्मंत्रिं0ं

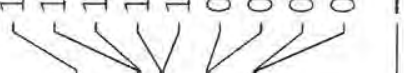

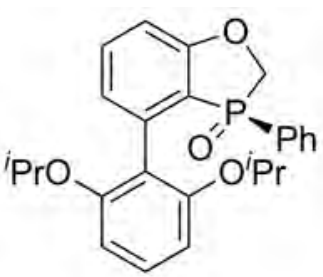

3k

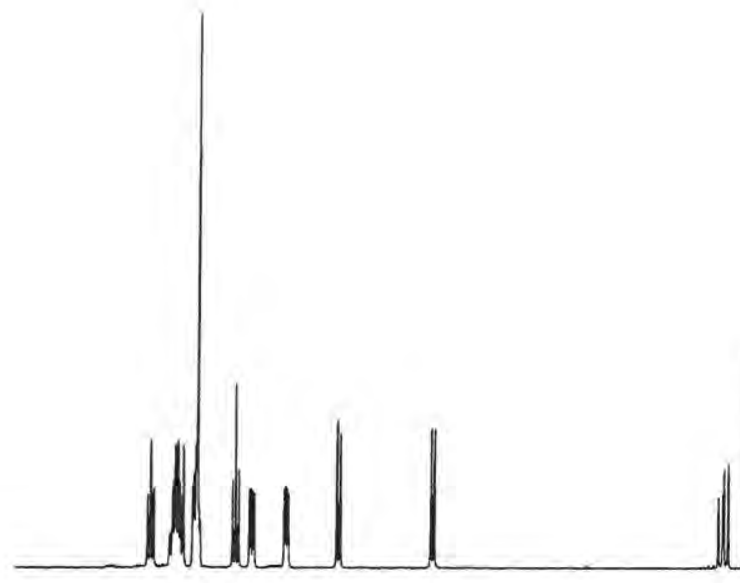

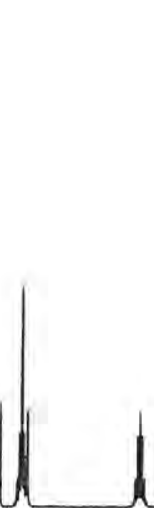

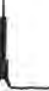

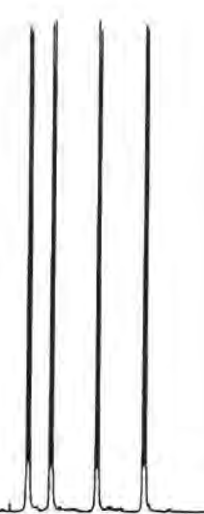

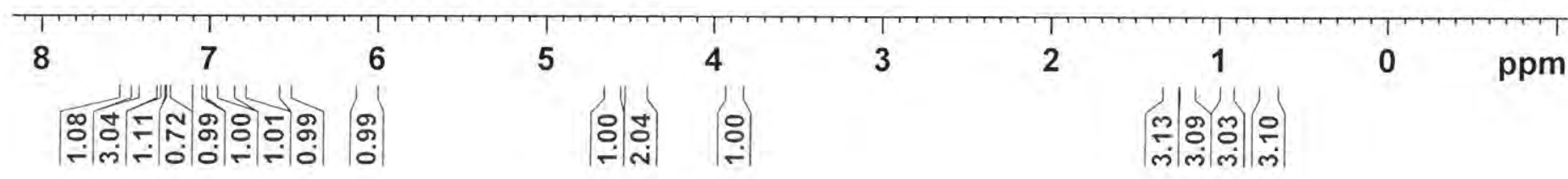

\section{Current Data Parameters NAME \\ EXPNO \\ 103669-044 \\ PROCNO}

F2 - Acquisition Parameters

Date_ 20151002

Time $\quad 12.26$

spect
PRSTRUM $5 \mathrm{~mm}$ PABBI $1 \mathrm{H}-$

PULPROG $\quad$ q $z g 10$

SOLVENT

NS

DS

SWH

EIDRES

$A Q$

RG

DW

$\mathrm{DE}$

TE

TDO

$$
\begin{array}{r}
\overline{3} 2768 \\
\text { CDC13 } \\
16 \\
0 \\
9014.423 \mathrm{~Hz} \\
0.275098 \mathrm{~Hz} \\
1.8175317 \mathrm{sec} \\
1440 \mathrm{usec} \\
55.467 \mathrm{usec} \\
6.50 \mathrm{usec} \\
299.0 \mathrm{~K} \\
2.0000000 \mathrm{sec}
\end{array}
$$

$========$ CHANNEL $\mathrm{f} 1$

NUC1

P1

PL1

PLIW$$
1
$$

$1 \mathrm{H}$ $3.00 \mathrm{~dB}$

$14.98121262 \mathrm{~W}$

$500.1334009 \mathrm{MHz}$

E2 - Processing parameters

SI 16384

$\begin{array}{lr}\text { SI } & 16384 \\ \text { SE } & 500.1300138 \mathrm{MHz}\end{array}$

WDW $\quad 500.1300138$

SSB 0

30

GB $\quad 0$

$0.30 \mathrm{~Hz}$

1.00 
103669-044 column

가의

n 舟 댁ㄷㄱㄷㅐ

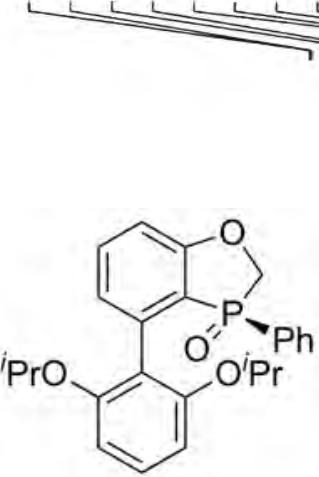

$3 k$

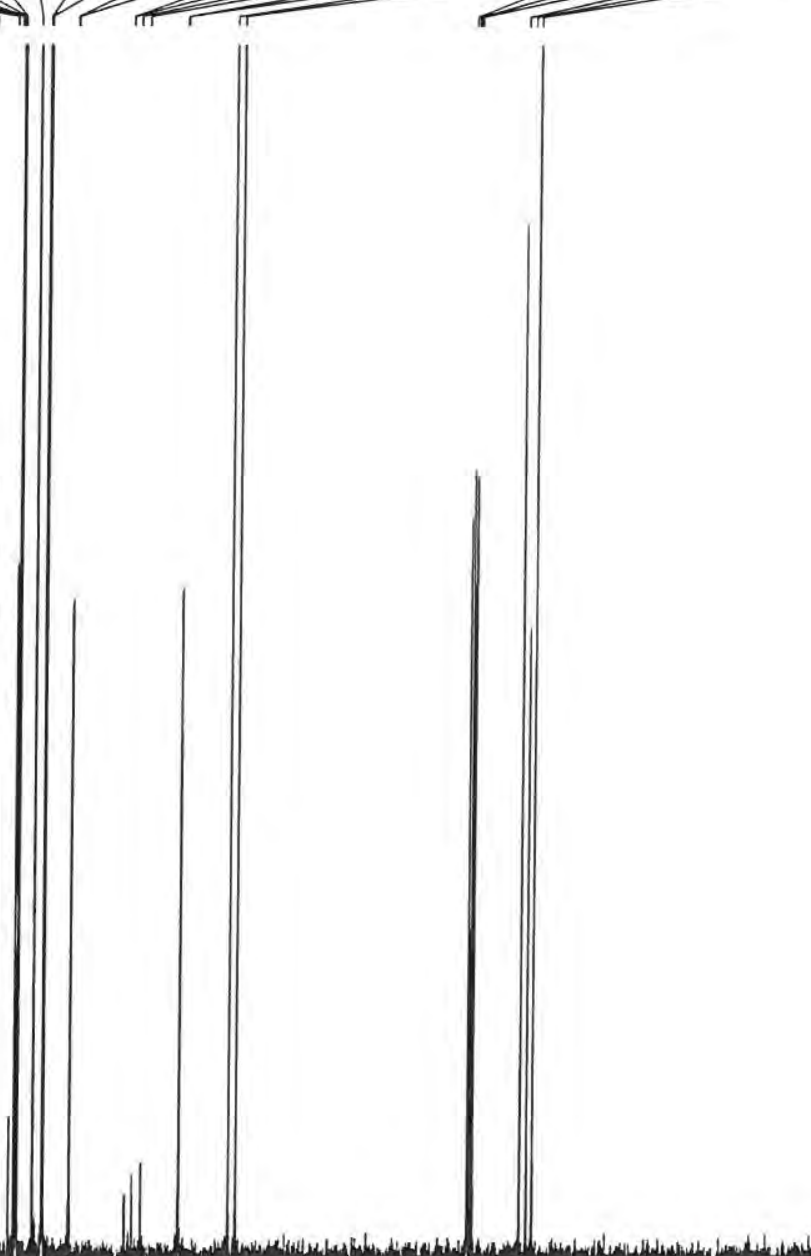

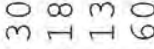

$\sim \sim \sim$

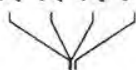

Current Data Parameters NAME 103669-044

PROCNO

F2 - Acquisition Parameter

Date_ 20151002

Time $\quad 16.25$

spect
INSTRUM

PULROG

SOLVENT

NS

SWH

FIDRES

AQ

RG

$\mathrm{DW}$

TE

D1

DEITA

TDO

SEO1

NUC

PIW1

$\mathrm{SFO} 2$

NUC2

CPDPRG [2

PCPD2

PIW2

PLW12

zgpg
32768

$\mathrm{CDC} 13$
226

$24038.461 \mathrm{~Hz}$

$0.733596 \mathrm{~Hz}$

$0.6815744 \mathrm{sec}$ 2050

20.800 usec 6.50 usec $298.0 \mathrm{~K}$

$2.00000000 \mathrm{sec}$ $0.03000000 \mathrm{sec}$ $1.89999998 \mathrm{sec}$ $100.6243395 \mathrm{MHz}$ $13 \mathrm{C}$ 10.10 usec $-1.00000000 \mathrm{~W}$ $400.1316005 \mathrm{MH}$

$1 \mathrm{H}$

$$
\text { waltz } 16
$$

90.00 usec

$-1.00000000 \mathrm{~W}$

$-1.0000000 \mathrm{~W}$

$-1.00000000 \mathrm{~W}$

F2 - Processing parameters

E2 - Processing parameter

$\begin{array}{lc}\text { SI } & 65536 \\ \text { SF } & 100.6127690 \mathrm{MHz}\end{array}$

$\begin{array}{lr}\text { SE } & 100.6127690 \\ \text { WDW } & \text { EM }\end{array}$ SSB

PC

$1.00 \mathrm{~Hz}$

1.40 


\section{9-043 column}

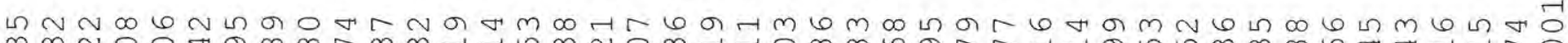
m $m$ NO

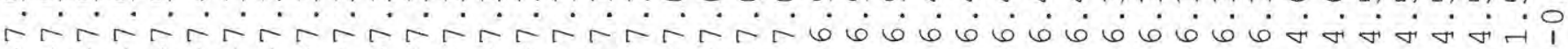
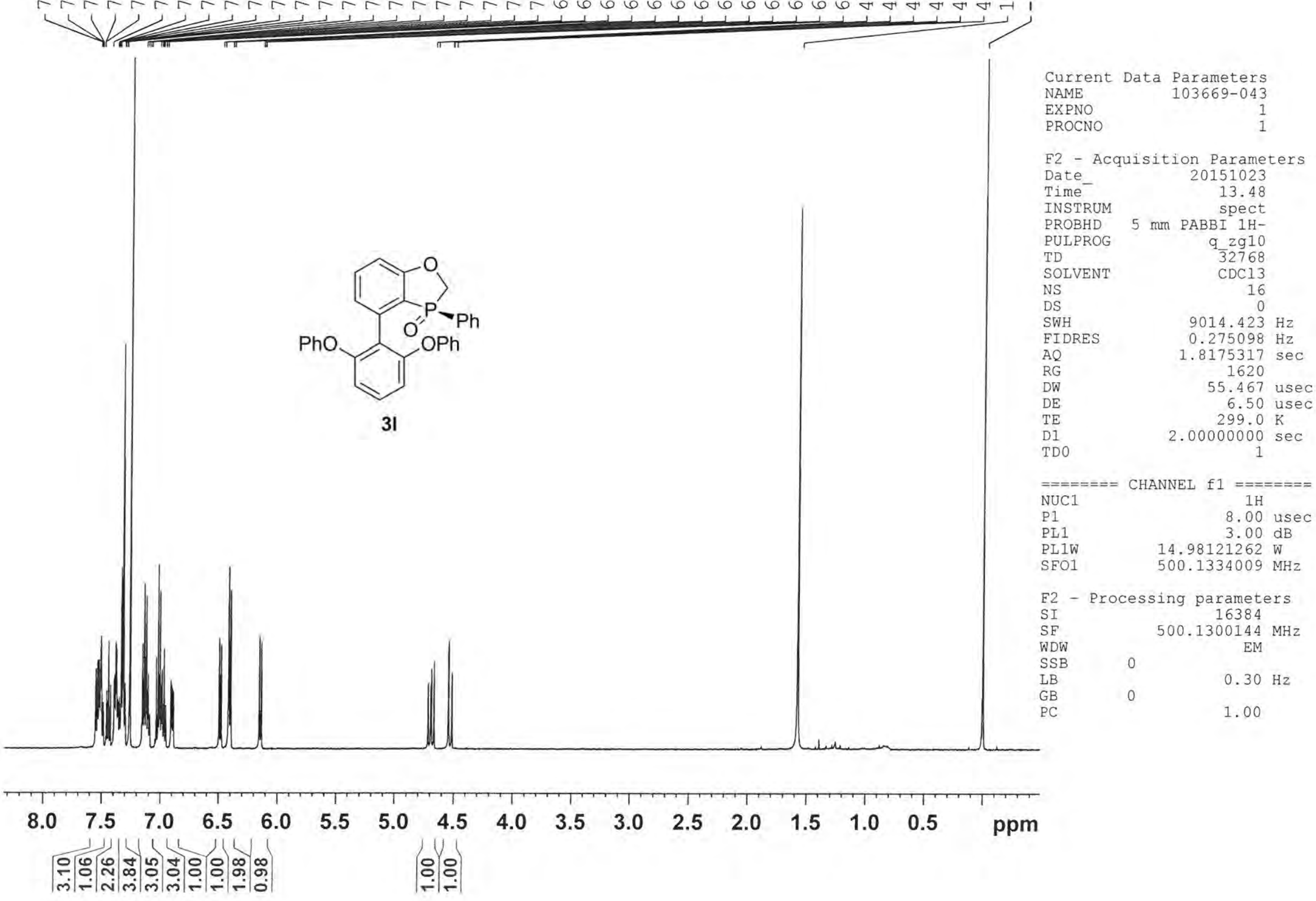


\section{$103669-043$}

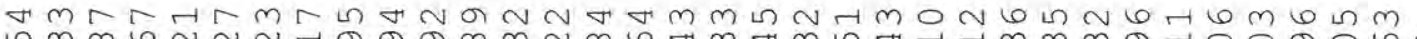

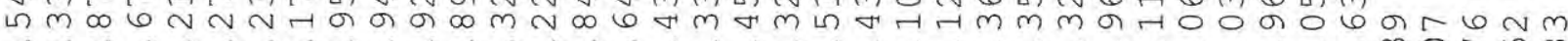

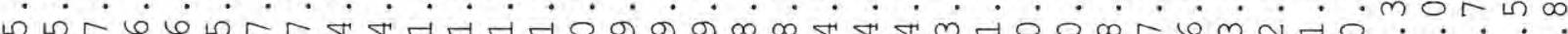

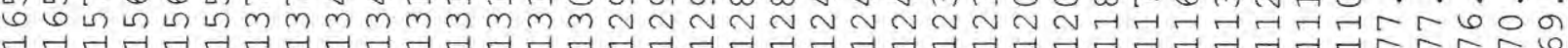

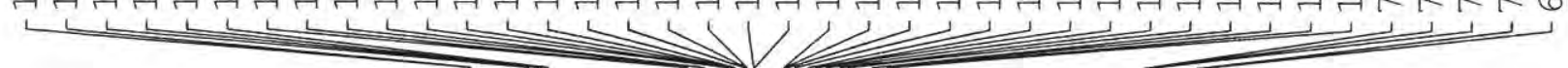

31
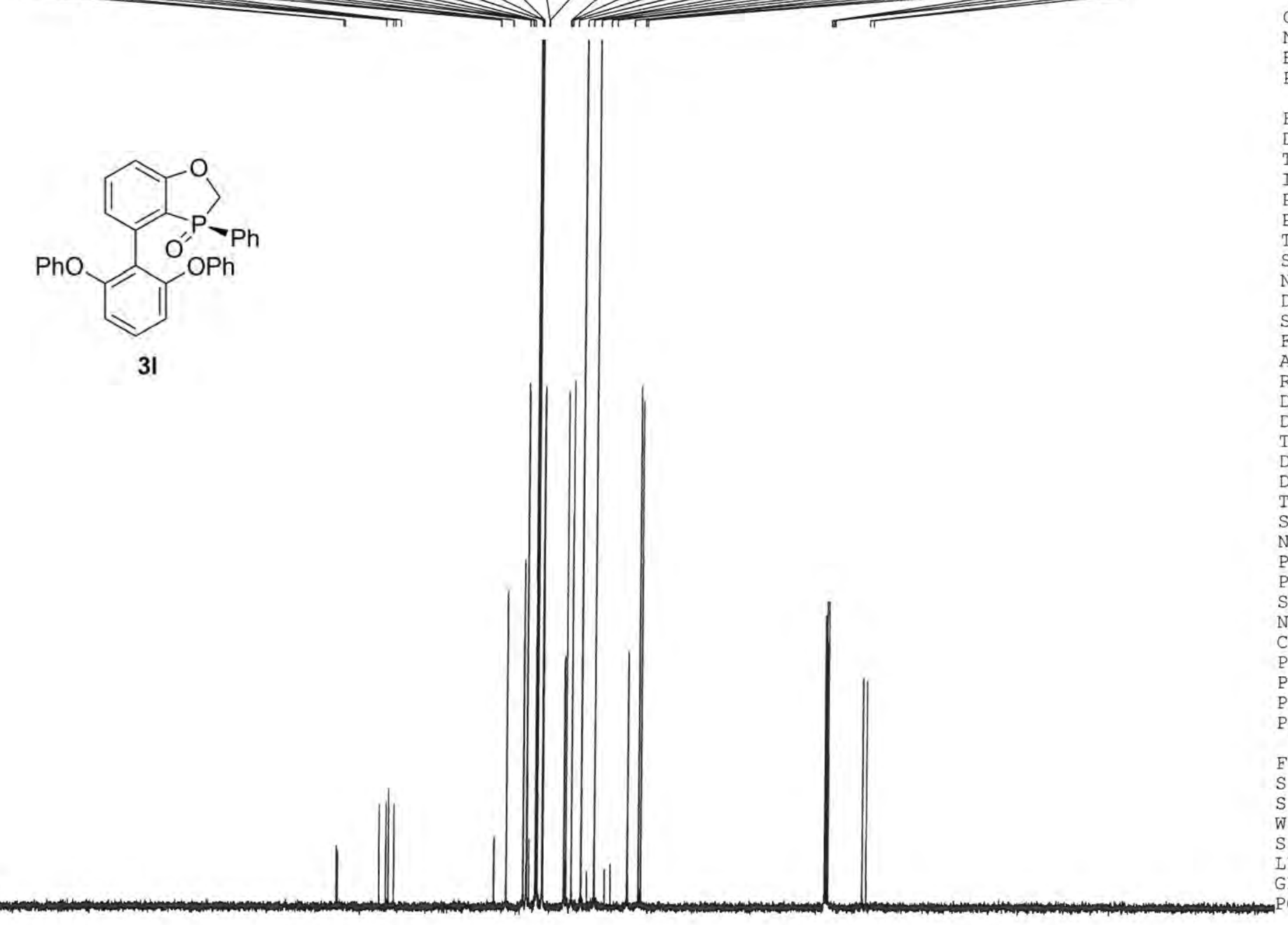

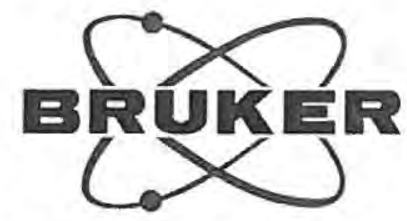

Current Data Parameters NAME EXPNO

F2 - Acquisition Parameters

Date_ 20151027

Time 16.52 h

INSTRUM

PROBHD

PULPROG

SOLVENT

NS

SWH

FIDRES

$A Q$
$R G$

RG

DW
DE
TE

DE

D1 1

TDO

SEO1

NUC1

PLW1

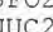

CPDPRG [ 2

PCPD2

PLW2

PLW12 2104450_0178! zgpg
32768
CDC13

512
4

$24038.461 \mathrm{~Hz}$ $0.733596 \mathrm{~Hz}$ 2050 20.800 usec 6.50 usec 2.00000000 sec $0.03000000 \mathrm{sec}$ 1 $100.6243395 \mathrm{MH}$ $13 \mathrm{C}$

10.00 usec $400.1316005 \mathrm{MHz}$

$$
\text { waltz } 16
$$

waltzl6
90.00 usec $12.05399990 \mathrm{~W}$ $12.05399900 \mathrm{~W}$ $0.15073690 \mathrm{~W}$

2 - Processing parameters I 65536 F $\quad 100.6127690 \mathrm{MH}$ SSB $\quad 0$ $\begin{array}{ll}\mathrm{LB} & 0 \\ \mathrm{~GB} & 0\end{array}$ EM

$1.00 \mathrm{~Hz}$ 1.40 


\section{9-046 column}

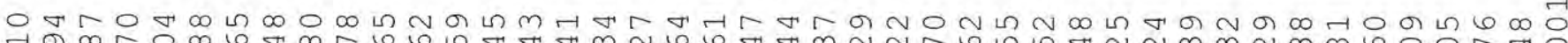
西至

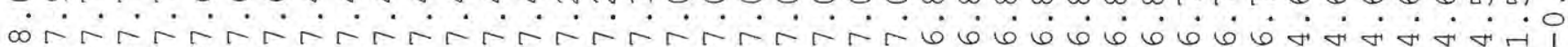
$\longrightarrow$

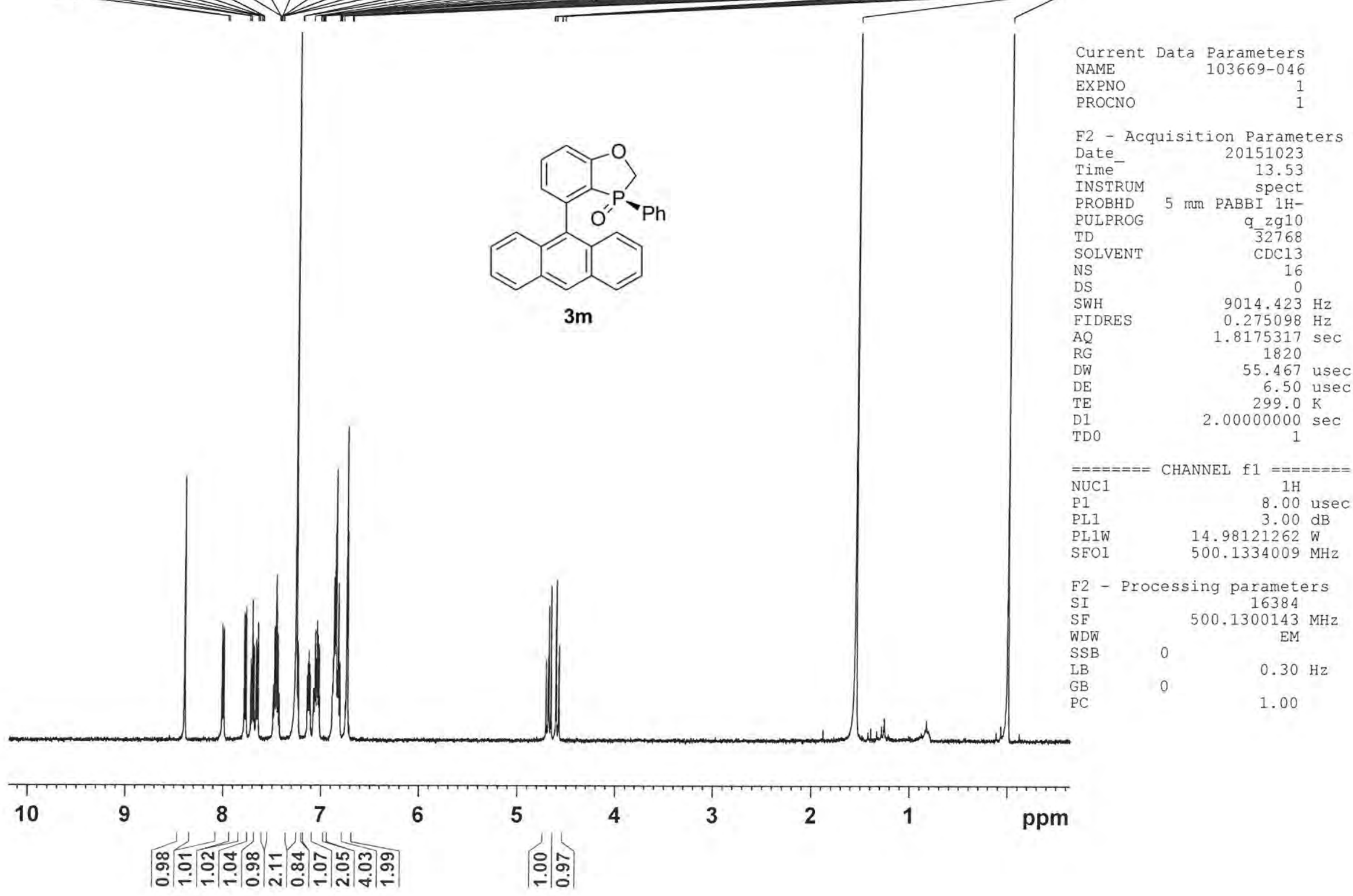




\section{9-046}

그는

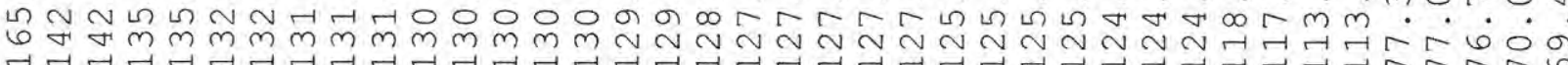

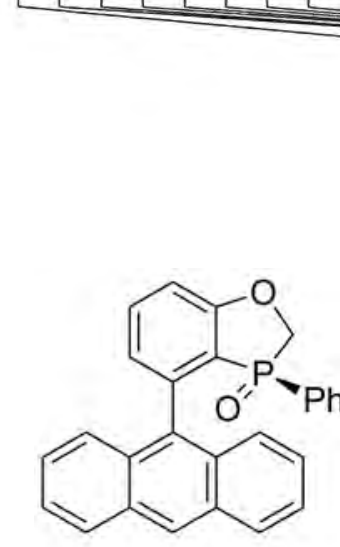

$3 m$
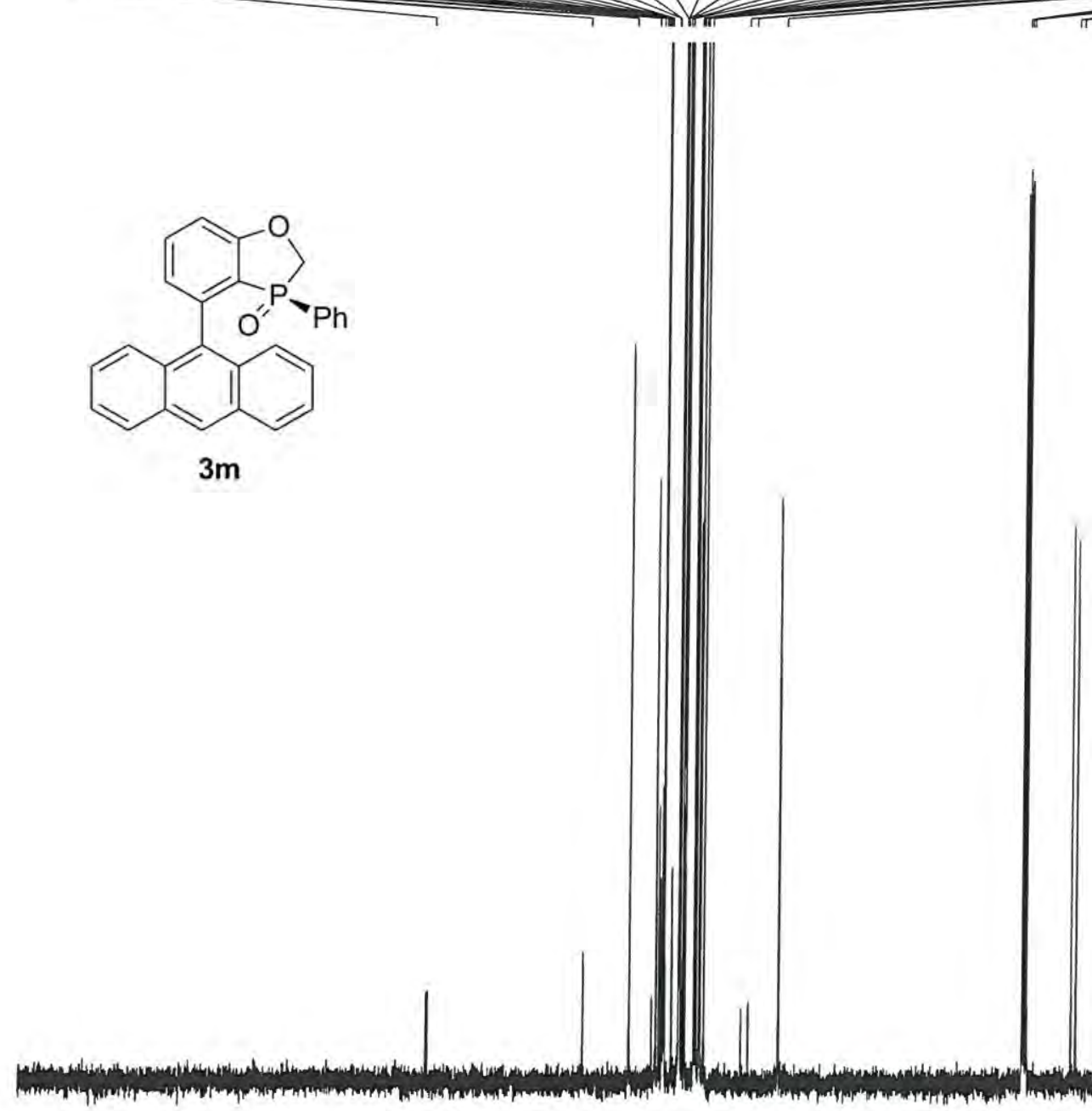

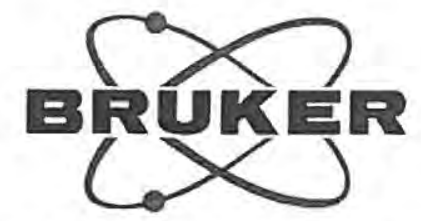

Current Data Parameters NAME 103669-046 PROCNO

F2 - Acquisition Parameters

Date_ 20151029

Time 11.44

INSTRUM SPect

PROBHD 2104450_0178

PULPROG
2gpg

TD 32768

DS

SWH

$24038.461 \mathrm{~Hz}$

$\quad 0.733596 \mathrm{~Hz}$

AQ

DW

DE

D1

D11

TD0
SEO1
NUC1

P1

PLW 1

SFO2

CPDPRG [ 2

PCPD2

PLW2

PLW12

$0.6815744 \mathrm{sec}$ 203

20.800 usec 6.50 use

$2.00000000 \mathrm{sec}$ 0.03000000 sec $100.6243395 \mathrm{MHz}$ $13 \mathrm{C}$

51.08800125 $400.1316005 \mathrm{MH}$ waltzl6 90.00 usec $12.05399990 \mathrm{~W}$ $.30075201 \mathrm{~W}$

F2 - Processing parameters $\begin{array}{lc}\text { SI } & 65536 \\ \text { SF } & 100.6127690 \mathrm{MHz}\end{array}$ WDW SSB LB 


\section{9-067 column}

r⿻ m $m$ m

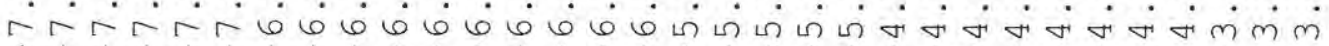

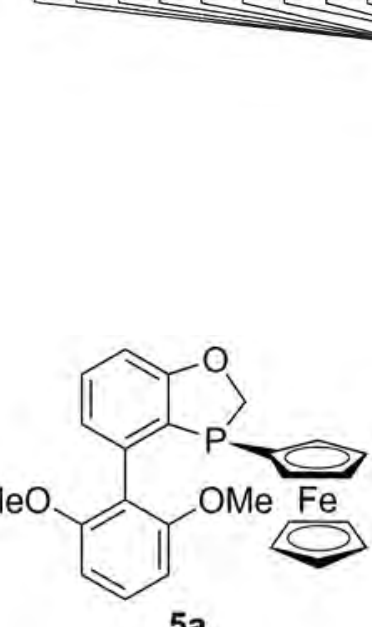

$5 a$

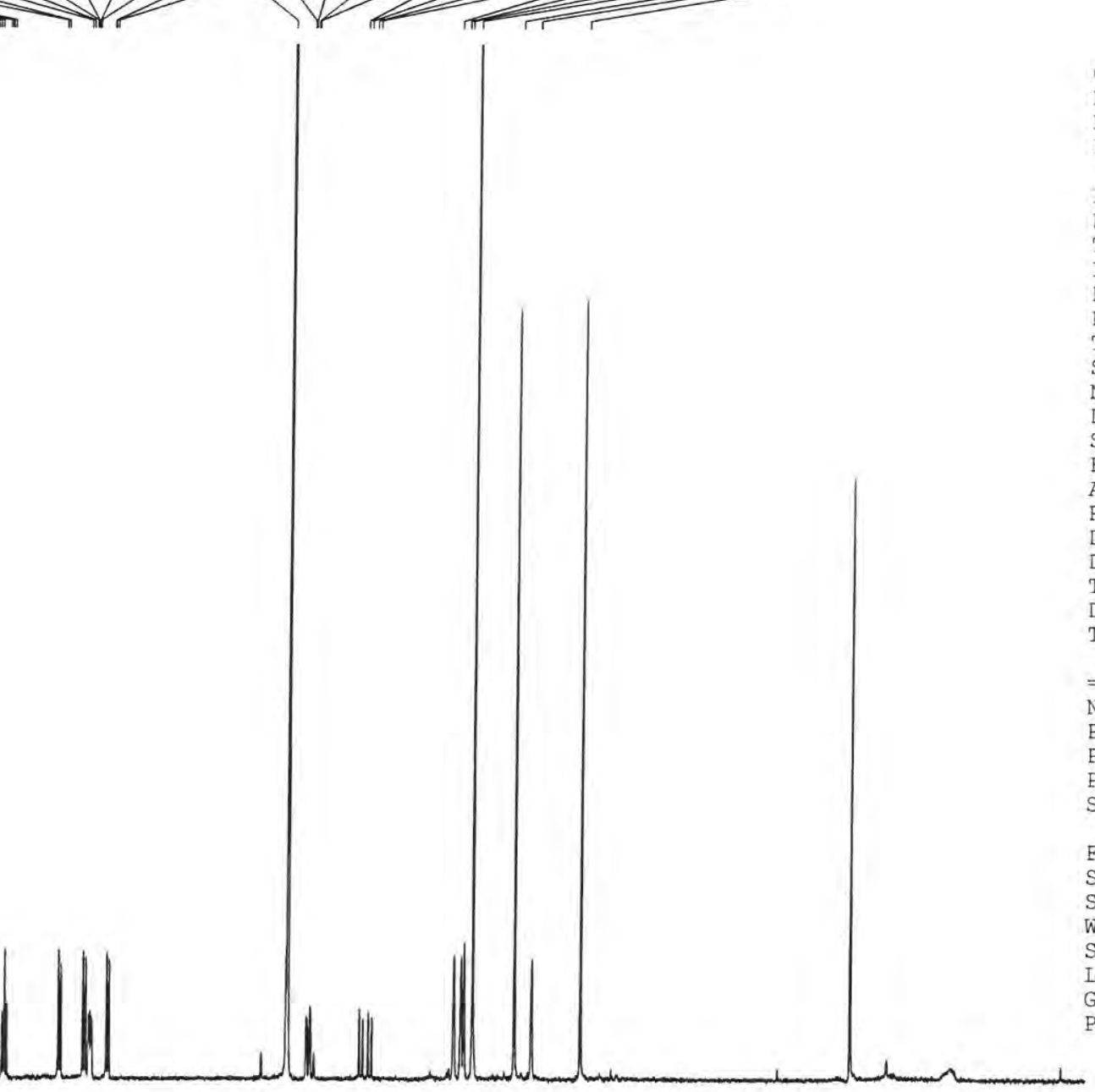

Current Data Parameter

NAME $103669-067$

EXNO

F2 - Acquisition Parameters

Date_ 20151019

Time $\quad 13.50$

spect
INSTRUM $5 \mathrm{~mm}$ PABBO BB

PROBHD $5 \mathrm{~mm}$ PABBO BB-

PULPROG व 2 Z10

$\mathrm{CD} 2 \mathrm{C} 12$

DS

SWH

FIDRES

AQ

DW

DE

D1

$9014.423 \mathrm{~Hz}$

$0.275098 \mathrm{~Hz}$

$1.8175317 \mathrm{sec}$

55.467 usec

6.50 usec $299.0 \mathrm{~K}$

$2.00000000 \mathrm{sec}$

$========$ CHANNEI $\mathrm{f} 1,=======$ NUC

P1
PL1

$18.43091774 \mathrm{~dB}$

$18.43091774 \mathrm{~T}$

$500.1334009 \mathrm{MHz}$

F2 - Processing parameters

I 16384

SF $\quad 500.1300146 \mathrm{MHz}$

SSB $\quad 0$

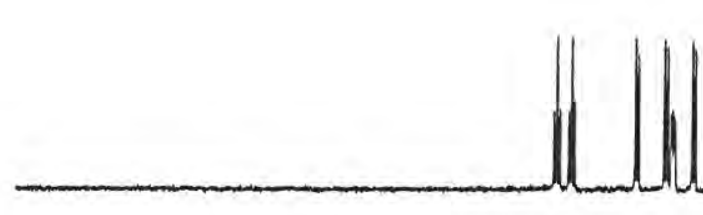

LB

$\mathrm{PC}$

$0.30 \mathrm{~Hz}$

1.00

8

7

6

5

4

3

2

1 ppm

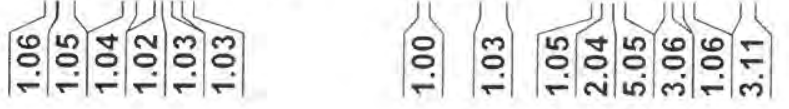




\section{$103669-067$}

$\nabla 6 \infty$ \% 6 म

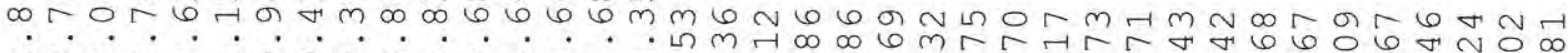

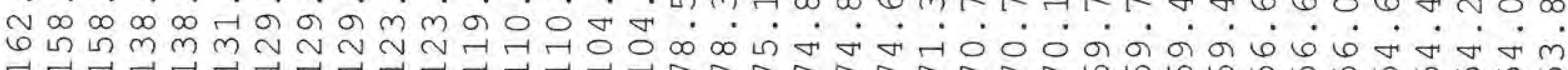

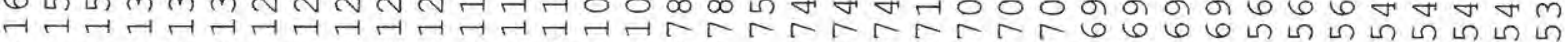

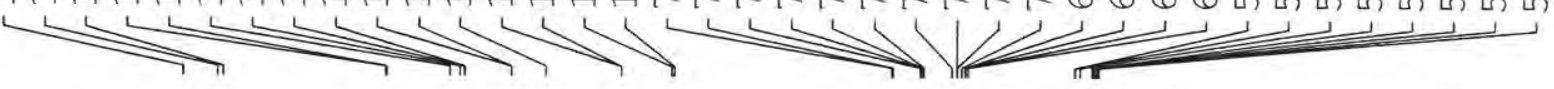

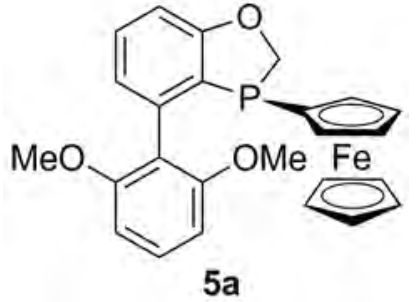

5 a
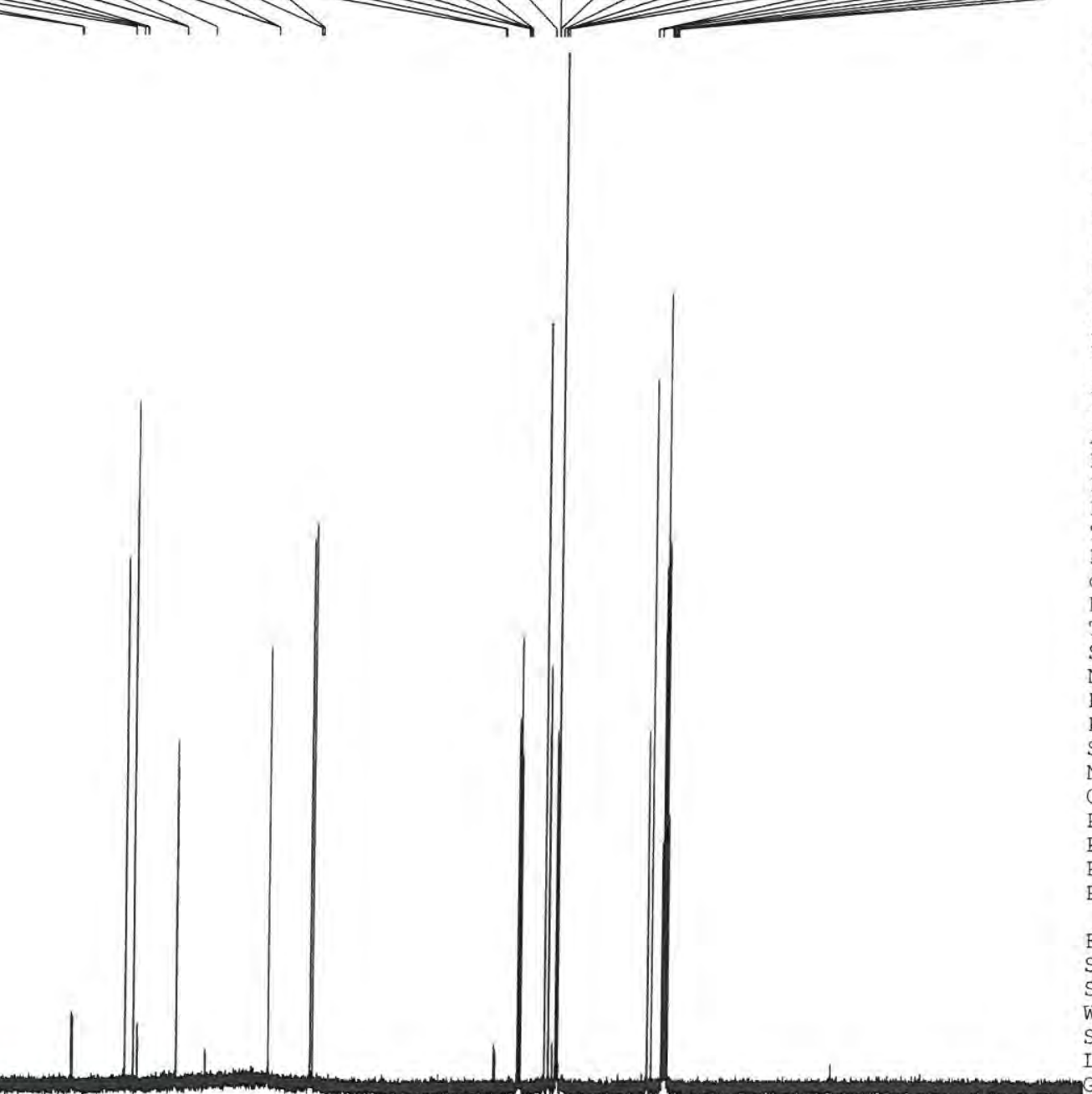

Current Data Parameters

NAME

EXPNO

F2 - Acquisition Parameters

Date_ 20151028

Time

INSTRUM

PROBHD

PULPROG

TD

SOLVENT

NS

DS

FIDRES

$A Q$
$R G$
$D W$

DW

TE

TE
D1 1

dII

DELTA

TDO

NEO1

SEC1

PLW1

SFO2

CPDPRG

PCPD2

PLW2

PLW12

PLW13

spect
$\mathrm{mm}$ PABBI $1 \mathrm{H}-$

zpg
1072

$\mathrm{CD} 2 \mathrm{C} 12$
5000

5000

$31250.000 \mathrm{~Hz}$ $2.0971520 \mathrm{sec}$ 2050

16.000 usec 6.50 usec

1.00000000

$000 \mathrm{sec}$

$0.89999998 \mathrm{sec}$

125.7698617

13

15.75 usec $500.1325007 \mathrm{WHz}$

$\mathrm{MHz}$ waltzit

80.00 usec

$-1.00000000 \mathrm{~W}$

$-1.00000000 \mathrm{~W}$

F2 - Processing parameters

SI 131072

$\begin{array}{lr}\text { SE } & 125.7576847 \mathrm{MHz} \\ \text { WDW } & \text { EM }\end{array}$

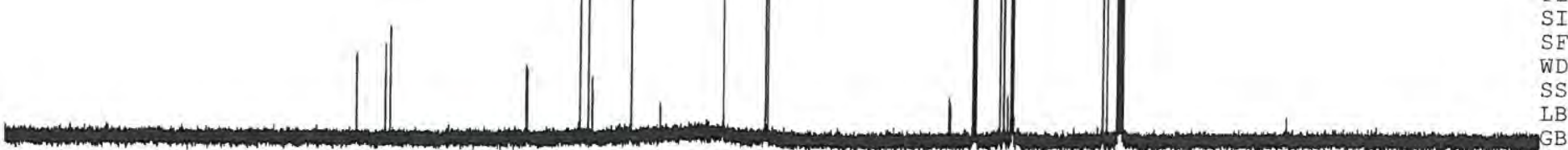

$0.30 \mathrm{~Hz}$

1.40 


\section{9-063 column}

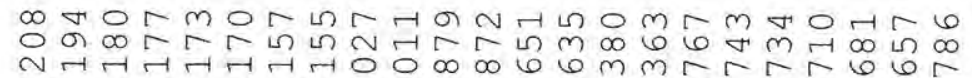

rarararara

$\longrightarrow$

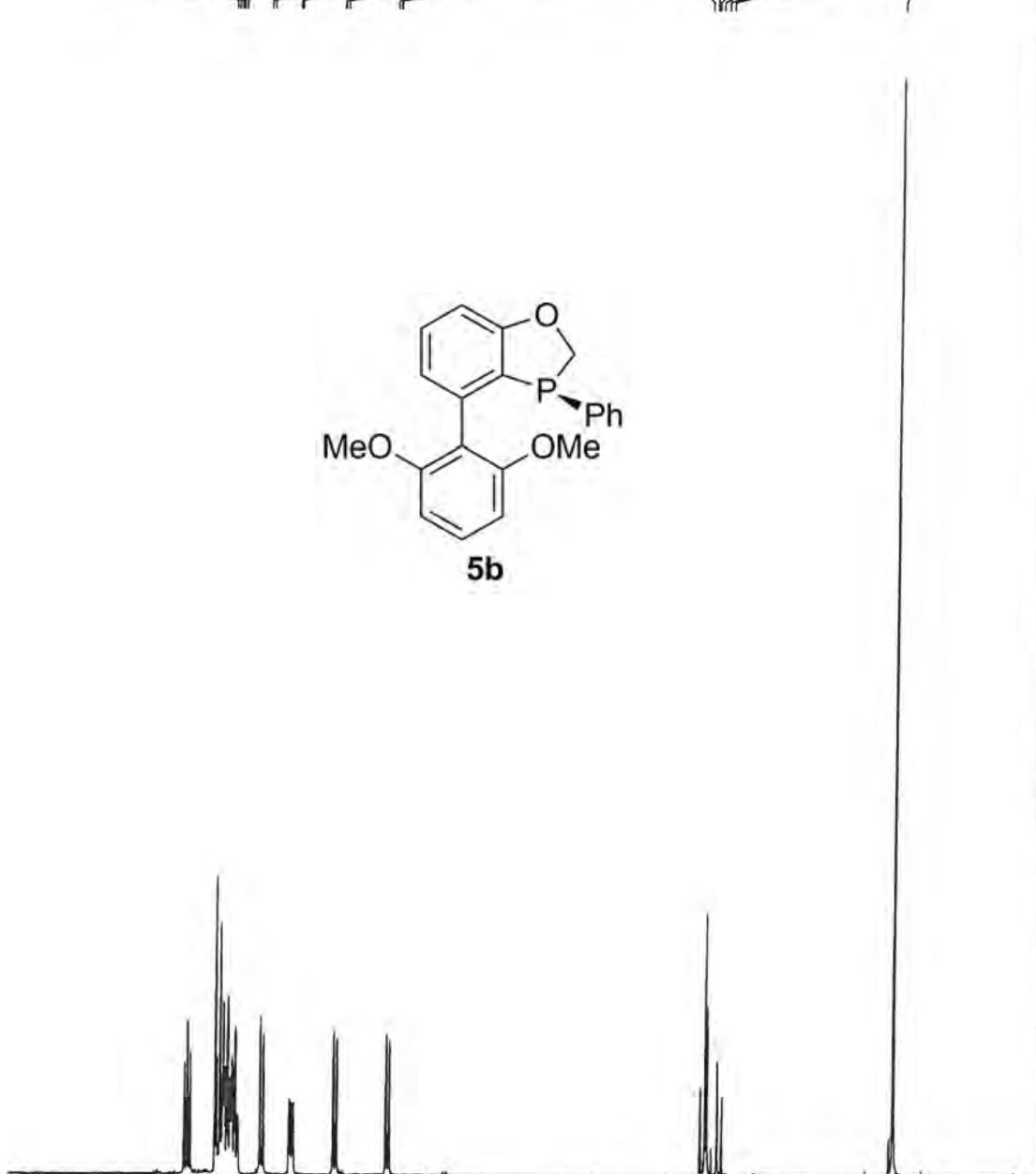

Current Data Parameters 103669-063 EXPNO

F2 - Acquisition Parameters

Date 20151015

Time 17.40

INSTRUM

PROBHD

$5 \mathrm{~mm}$ PABBO BB-

PULPROG

SD

NS

DS

SWH

EIDRES

AQ

RG

$D W$

TE

D1
q 2910
$\overline{3} 2768$
$9014.423 \mathrm{~Hz}$
$1.8175317 \mathrm{sec}$
512
55.467 usec 6.50 usec $299.0 \mathrm{~K}$

16
0

$2.00000000 \mathrm{sec}$

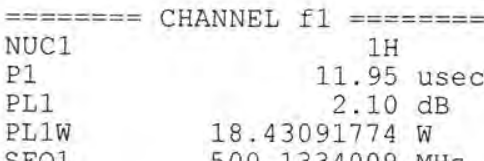

$\begin{array}{ll}\text { PL1W } & 18.43091774 \mathrm{~W} \\ \text { SFO1 } & 500.1334009\end{array}$

F2 - Processing parameters

SI Processing parameter

$\begin{array}{lr}\text { SI } & 16384 \\ \text { SF } & 500.1300144 \mathrm{MHz}\end{array}$

SE

$500.1300144 \mathrm{MHz}$

$\begin{array}{lll}\text { LB } & 0 & 0.30 \mathrm{~Hz}\end{array}$
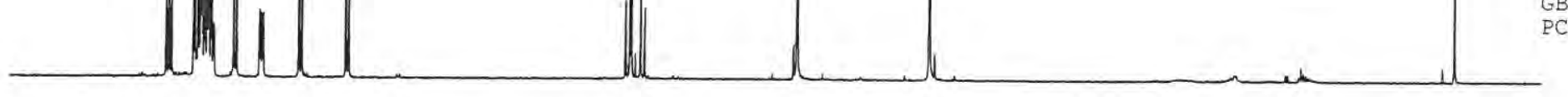

PC

1.00

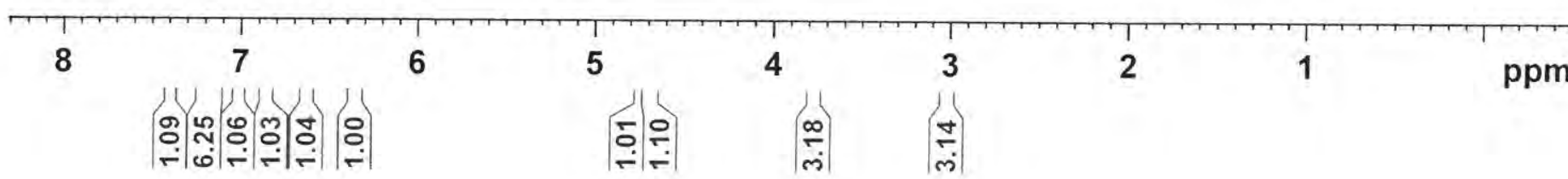




\section{9-063 column}

이맙

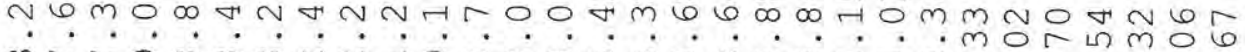

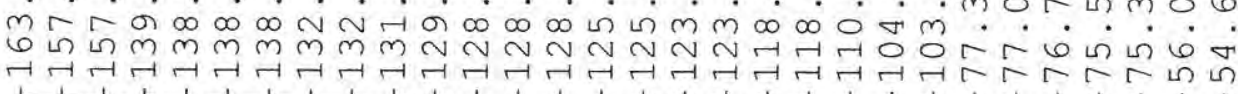

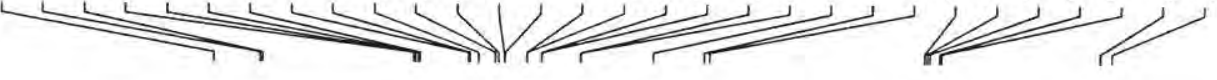

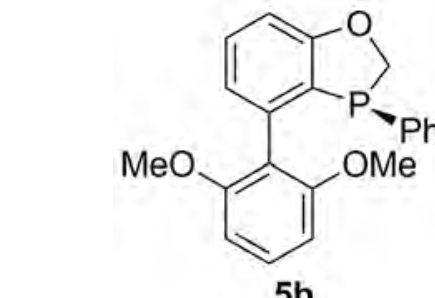

$5 b$

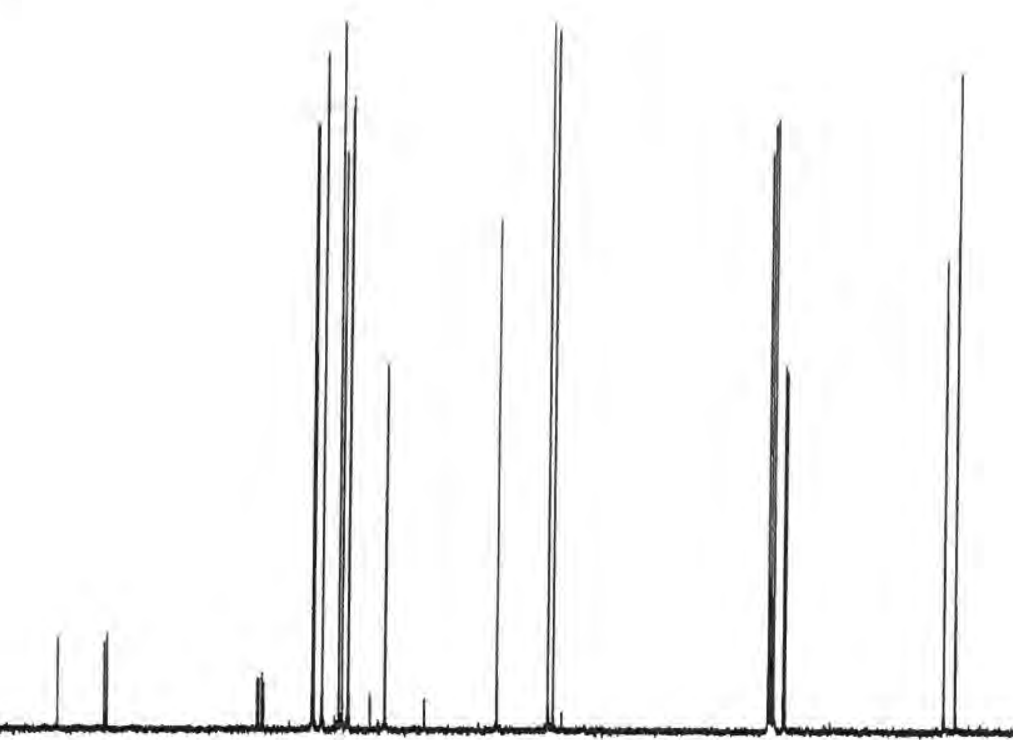

Current Data Parameters

EXPNO

F2 - Acquisition Parameters

Date_ 20151017

Time

INSTRUM

PROBHD

PULPROG

SOLVENT

NS

DS
SWH

FIDRES

AQ

RG

DW

DE

TE

d11

DELTA

TDO

SEO1

NUC1

P1

PIW 1

SEO2

NUC2

CPDPRG [ 2

PCPD2

PIW2

PIN

PLW12
PLW13

In PABBO BB-

$$
\text { BO } \mathrm{BB}-
$$

zgpg
32768

CDC13

8000

$24038.461 \mathrm{~Hz}$ $0.733596 \mathrm{~Hz}$ $0.6815744 \mathrm{sec}$ 2050

20.800 usec 6.50 usec $298.0 \mathrm{~K}$

$2.00000000 \mathrm{sec}$ $0.03000000 \mathrm{sec}$ $1.89999998 \mathrm{sec}$ $100.6243395 \mathrm{MHz}$ $13 \mathrm{C}$ -1.000000 usec $400.1316005 \mathrm{MH}$ waltz 16 waltzi6 $-1.00000000 \mathrm{~W}$ $-1.000000$ -1.00000000 W

F2 - Processing parameters SI 65536 SF $\quad 100.6127690 \mathrm{MH}$ SSB $\quad 0$ 0 


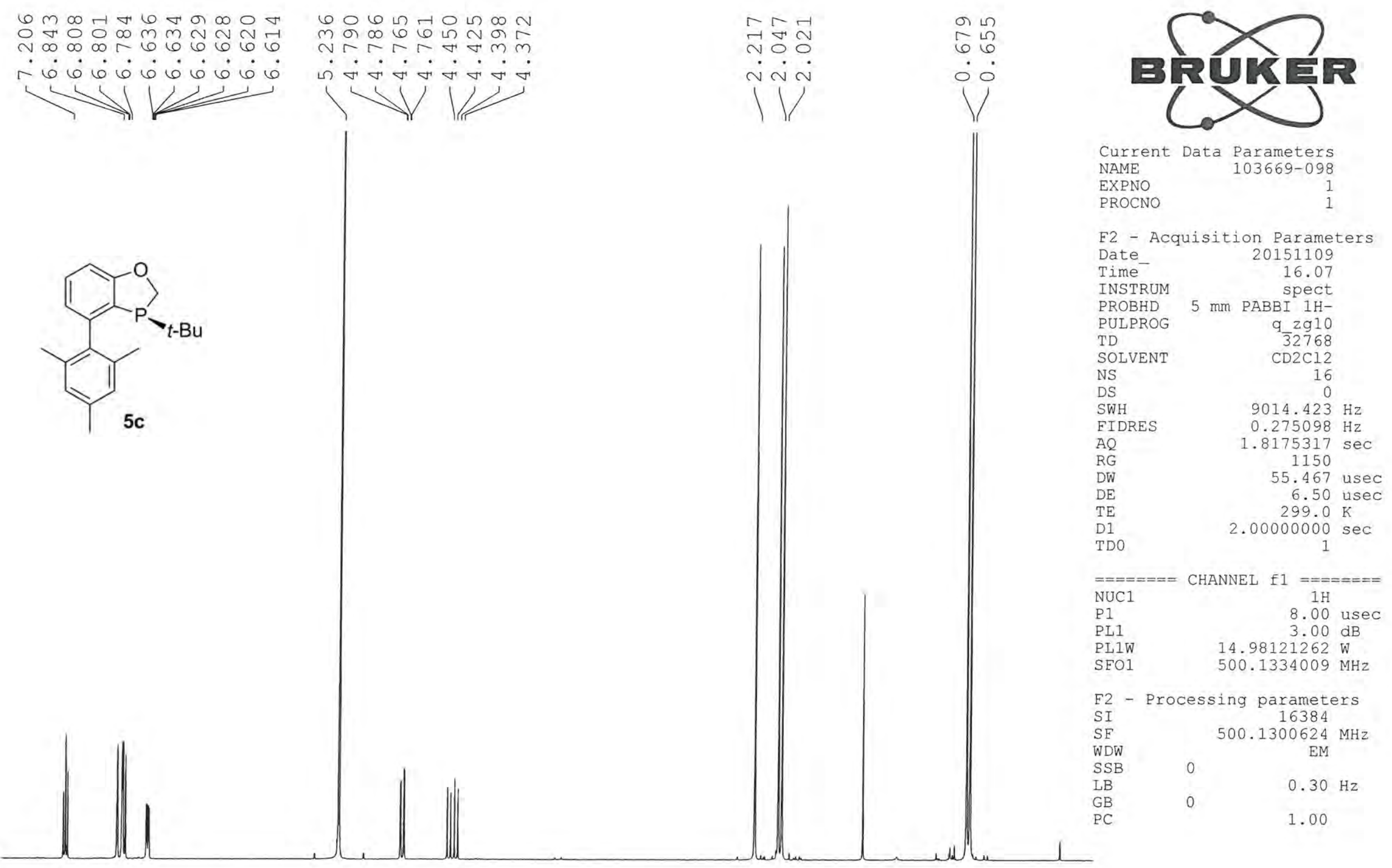

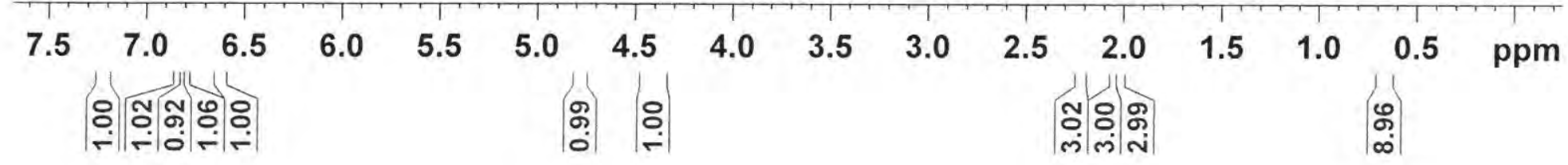




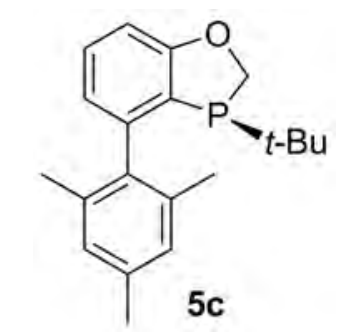

থ

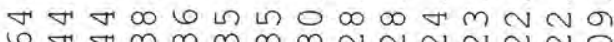
$6 \sigma \sigma m m m m \sim \sim \sim \sim N 0$

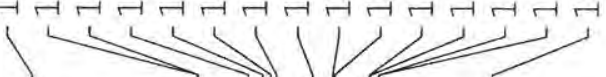

भ

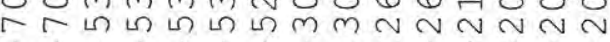
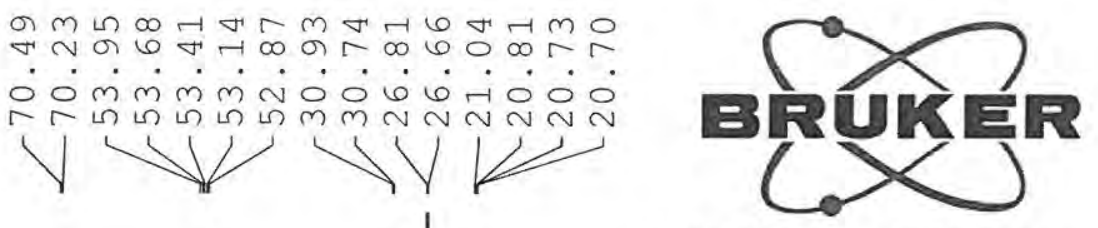

Current Data Parameters NAME 103669-099

EXPNO

F2 - Acquisition Parameters

Date_ 20151111

Time $18.15 \mathrm{~h}$

INSTRUM spect

PROBHD Z104450 0178

PULPROG Zgpg

SOLVENT

NS

DS

$\begin{array}{lr}\text { SWH } & 24038.461 \mathrm{~Hz} \\ \text { FIDRES } & 0.733596 \mathrm{~Hz}\end{array}$

$0.6815744 \mathrm{sec}$

RG

RG

$D W$
$D E$
TE
$D 1$

D1

D11

SFO1

NUC1

PI

PLW1

SFO2

CPDPRG

CPDPR

PCPD2

PLW 2

PLW13

203

20.800 usec 6.50 use

$2.00000000 \mathrm{sec}$

$0.03000000 \mathrm{sec}$

$100.6243395 \mathrm{MHz}$

$13 \mathrm{C}$ usec
10.00 usec

$51.08800125 \mathrm{~W}$

$400.1316005 \mathrm{MHz}$

$1 \mathrm{H}$

$$
\text { waltz } 16
$$

90.00 usec

$12.05399990 \mathrm{~W}$

$0.15073690 \mathrm{~W}$

F2 - Processing parameters

SI 65536

SF $\quad 100.6127690 \mathrm{MHz}$

WDW

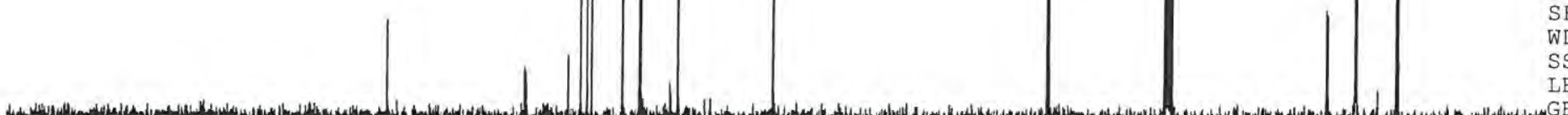

$\begin{array}{ll}0 & 1.00 \mathrm{~Hz} \\ 0 & 1.40\end{array}$

\begin{tabular}{llllllllllll}
\hline 200 & 180 & 160 & 140 & 120 & 100 & 80 & 60 & 40 & 20 & $\mathrm{ppm}$
\end{tabular}

